\title{
NONLINEAR OBLIQUE BOUNDARY VALUE PROBLEMS FOR NONLINEAR ELLIPTIC EQUATIONS
}

\author{
GARY M. LIEBERMAN AND NEIL S. TRUDINGER
}

\begin{abstract}
We consider the nonlinear oblique derivative boundary value problem for quasilinear and fully nonlinear uniformly elliptic partial differential equations of second order. The elliptic operators satisfy natural structure conditions as introduced by Trudinger in the study of the Dirichlet problem while for the boundary operators we formulate general structure conditions which embrace previously considered special cases such as the capillarity condition. The resultant existence theorems include previous work such as that of Lieberman on quasilinear equations and Lions and Trudinger on Neumann boundary conditions.
\end{abstract}

1. Introduction. In this paper we are concerned with oblique, nonlinear elliptic boundary value problems of the general form,

$$
\begin{aligned}
& F[u]=F\left(x, u, D u, D^{2} u\right)=0 \quad \text { in } \Omega, \\
& G[u]=G(x, u, D u)=0 \quad \text { on } \partial \Omega,
\end{aligned}
$$

where $\Omega$ is a bounded smooth domain in Euclidean $n$-space, $\mathbf{R}^{n}$, and $F$ and $G$ are real valued functions on the domains $\Gamma=\Omega \times \mathbf{R} \times \mathbf{R}^{n} \times \mathbf{S}^{n}, \Gamma^{\prime}=\partial \Omega \times \mathbf{R} \times \mathbf{R}^{n}$. Here $\mathbf{S}^{n}$ denotes the $n(n+1) / 2$ dimensional linear space of $n \times n$ real symmetric matrices, and $D u=\left(D_{i} u\right)$, and $D^{2} u=\left[D_{i j} u\right]$ denote the gradient and Hessian matrix of the real valued function $u$. By a classical solution of (1.1), (1.2) we shall mean a function $u \in C^{1}(\bar{\Omega}) \cap C^{2}(\Omega)$ satisfying equations (1.1), (1.2) in a pointwise sense.

Letting $X=(x, z, p, r), X^{\prime}=(x, z, p)$ denote points in $\Gamma, \Gamma^{\prime}$, we shall adopt the following definitions of ellipticity and obliqueness for functions $F, G$ differentiable with respect to $r, p$ respectively. Namely, the operator $F$ is elliptic at $X \in \Gamma$ if the matrix $F_{r}=\left[F^{i j}\right]=\left[\partial F / \partial r_{i j}\right]$ is positive at $X$; while the operator $G$ is oblique at $X^{\prime} \in \Gamma^{\prime}$ if $\chi=G_{p_{\gamma}}=G_{p} \cdot \gamma$ is positive at $X^{\prime}$, where $\gamma$ is the unit inner normal to $\partial \Omega$. Letting $\lambda, \Lambda$ denote the minimum and maximum eigenvalues of $F_{r}$, we shall call $F$ uniformly elliptic with respect to some subset $\mathcal{U} \subset \Gamma$ if the ratio $\Lambda / \lambda$ is bounded on $U$.

We shall treat here uniformly elliptic operators $F$ and oblique operators $G$ subject to certain natural structure conditions. For the operators $F$ these conditions were introduced by Trudinger [30] as an extension to fully nonlinear operators of the natural conditions of Ladyzhenskaya and Ural'tseva [14] for uniformly elliptic quasilinear operators. Letting $\mu, \mu_{0}, \mu_{1}, \mu_{2}$ denote nondecreasing real functions, we may express these conditions as follows:

F1: $\Lambda \leq \lambda \mu(|z|)$ (Uniform ellipticity);

F2: $|F(x, z, p, 0)| \leq \lambda \mu_{0}(|z|)\left(1+|p|^{2}\right)$;

Received by the editors September 27, 1984.

1980 Mathematics Subject Classification. Primary 35J60, 35J65; Secondary 35B45. 
F3: $(1+|p|)\left|F_{p}\right|,\left|F_{z}\right|,\left|F_{x}\right| \leq \lambda \mu_{1}(|z|)\left(1+|p|^{2}+|r|\right)$;

F4: $(1+|r|)\left|F_{r x}\right|,(1+|r|)\left|F_{r z}\right|,(1+|r|)\left|F_{r p}\right|,\left|F_{p p}\right|,\left|F_{p z}\right|$, $\left|F_{p x}\right|,\left|F_{z z}\right|,\left|F_{z x}\right|,\left|F_{x x}\right| \leq \lambda \mu_{2}(|z|+|p|)(1+|r|)$;

F5: $F_{r r} \leq 0$ (that is, $F$ is concave with respect to $r$ ),

for all $X=(x, z, p, r) \in \Gamma$. Unless otherwise indicated the arguments of all functions occurring above are $(x, z, p, r)$. For oblique boundary operators $G$ we formulate corresponding conditions:

G2: $\left|G\left(x, z, p^{\prime}\right)\right| \leq \chi \mu_{0}(|z|)\left(1+\left|p^{\prime}\right|\right)$

G3: $(1+|p|)\left|G_{p}\right|,\left|G_{z}\right|,\left|G_{x}\right| \leq \chi \mu_{1}(|z|)(1+|p|)$,

for all $X^{\prime}=(x, z, p) \in \bar{\Omega} \times \mathbf{R} \times \mathbf{R}^{n}$, where $p^{\prime}=p-(p \cdot \gamma) \gamma$ is the tangential projection of $p$ and the normal vector field $\gamma$ and boundary function $G$ have been appropriately extended to $\bar{\Omega} \times \mathbf{R} \times \mathbf{R}^{n}$.

The conditions G2, G3 embrace a large class of boundary value problems including quasilinear oblique derivative problems of the form

$$
G[u]=b(x, u) \cdot D u+g(x, u)=0 \quad \text { on } \partial \Omega,
$$

and the capillarity boundary condition

$$
G[u]=\gamma \cdot D u-g(x, u) \sqrt{1+|D u|^{2}}=0 \text { on } \partial \Omega,
$$

where the contact angle, $\theta=\operatorname{arc} \cos g$, between the graph of $u$ and the cylinder $\partial \Omega \times \mathbf{R}$ in $\mathbf{R}^{n+1}$ is prescribed. Problem (1.3) is oblique when $b \cdot \gamma>0$, while problem (1.4) is oblique for $|g|<1$.

The method of continuity, as presented for example in [9], reduces the classical solvability of the boundary value problem (1.1), (1.2) to the establishment of a priori estimates in the Banach space $C^{2, \alpha}(\bar{\Omega})$ for some $\alpha>0$, for solutions of a family of related problems. For boundary value problems subject to the above natural structure conditions, we shall prove the following estimate.

THEOREM 1.1. Let $\Omega$ be a bounded domain in $\mathbf{R}^{n}$ with boundary $\partial \Omega \in C^{4}$ and suppose that $F \in C^{2}(\bar{\Gamma}), G \in C^{2}\left(\Gamma^{\prime}\right)$ satisfy the structure conditions $\mathrm{F} 1, \mathrm{~F} 2, \mathrm{~F} 3, \mathrm{~F} 4$, F5, G2, G3. Then if $0<\alpha<1$ and $u \in C^{2}(\bar{\Omega})$ is a solution of the boundary value problem $F[u]=0$ in $\Omega, G[u]$ on $\partial \Omega$, we have the estimate

$$
|u|_{2, \alpha ; \Omega} \leq C,
$$

where $C$ depends on $n, \mu, \mu_{0}, \mu_{1}, \mu_{2}, \alpha, \sup _{\Omega}|u|, \Omega$ and all the second derivatives of the functions $F$ and $G$.

As an example of the application of Theorem 1.1, we have the following existence theorem.

COROLlaRY 1.2. Let $\Omega, F$ and $G$ satisfy the hypotheses of Theorem 1.1, together with the conditions

$$
\sup _{\Gamma} F_{z}<0, \quad \sup _{\Gamma^{\prime}} G_{z} \leq 0 .
$$

Then there exists a unique classical solution $u$ of the boundary value problem $F[u]=$ 0 in $\Omega, G[u]=0$ on $\partial \Omega$ with $u \in C^{2, \alpha}(\bar{\Omega})$ for all $\alpha<1$.

Theorem 1.1 and Corollary 1.2 are new, even in the quasilinear case where they extend previous work of Lieberman [18]. In this case solvability of the boundary 
value problem (1.1) arises from only $C^{1, \alpha}(\bar{\Omega})$ estimates. Whereas the Dirichlet problem had been extensively studied for quasilinear equations $[\mathbf{9}, \mathbf{1 4}]$, the study of oblique boundary value problems had primarily focussed on conormal derivative problems for divergence form equations, such as the capillarity problem $[\mathbf{7}, \mathbf{1 4}, \mathbf{1 7}$, 32].

The $C^{2, \alpha}(\bar{\Omega})$ estimate of Theorem 1.1 is the culmination of several component estimates of lower order which are established separately in the different sections of this paper. In particular, in $\S 2$, we derive global Hölder estimates for solutions of (1.1), using a modification of the weak Harnack inequality [29]. In $\S 3$ we treat gradient bounds, obtaining gobal gradient estimates under the structure conditions F1, F2, F3, G2, G3, analogous to those for the Dirichlet problem [30]. Hölder estimates for derivatives are deduced in $\S 4$. These had been previously obtained for quasilinear equations by Lieberman [18] using different methods. In $\S 5$ we treat global bounds for second derivatives. Here the approach splits naturally into two stages. First is the derivation of one-sided estimates for pure tangential derivatives which has features in common with the Neumann type problems for the Bellman equation treated by Lions and Trudinger [27], as well as earlier work on the Dirichlet problem $[\mathbf{2 5}, \mathbf{1 1}]$. The second stage is the estimation of the pure normal second derivatives which involves substantially different arguments from the more standard devices, used for example in [27]. In $\S 6$ we finally reach the global Hölder estimates for second derivatives, employing a different and more general argument to that of $[\mathbf{2 7}]$, which like the first derivative estimates in [18] is based on conormal divergence structure inequalities. In the last section, we discuss the application of the preceding estimates to the boundary value problem (1.1), (1.2), where an alternate approach to the method of continuity, due to Lieberman $[\mathbf{1 6}$, 19], may also be used.

To conclude this introduction we remark that many of our estimates remain valid under more general structural hypotheses than F1, F2, F3, F4, F5 and certain extensions will be at least indicated in the course of this paper. In particular a mild nonuniformity may often be achieved by expressing structural conditions in terms of the Bernstein function $\mathcal{E}=F^{i j} p_{i} p_{j}$, and its second order extension $\mathcal{E}_{2}$, given by $\mathcal{E}_{2}=F^{i j} r_{i k} r_{j k}$. Also all notation, unless otherwise specified, will follow the book $[\mathbf{9}]$.

We wish to express our thanks to P.-L. Lions whose contribution in [27] stimulated our joint investigations. This research was carried out while we were both visiting Indiana University in the Fall of 1983 and we thank in particular Roger Newton and Bill Ziemer for their encouragement and hospitality as well as our home institutions for supporting our leave programmes.

2. Weak Harnack inequalities and Hölder estimates for solutions. Our Hölder estimates will all be derived from the weak Harnack inequality, Lemma 2.1 below. Its proof uses the corresponding interior estimate of Trudinger $[\mathbf{2 9}$, Theorem 9] (which is based on estimates of Krylov and Safonov [13]) and a comparison argument inspired by a result of Krylov [12, Lemma 2.1]. The latter was used by him to obtain boundary Hölder estimates on second derivatives of solutions of the Dirichlet problem. 
In order to state our result conveniently, we introduce the sets

$$
\begin{array}{ll}
B^{+}=\left\{x \in \mathbf{R}^{n}|| x \mid<1, x^{n}>0\right\}, & B^{0}=\left\{x \in \mathbf{R}^{n}|| x \mid<1, x^{n}=0\right\}, \\
G(\rho, R)=\left\{\left|x^{\prime}\right|<R, 0<x^{\prime \prime}<\rho R\right\}, & G^{\prime}(\rho, R)=\left\{\left|x^{\prime}\right|<R, \rho R<x^{n}<3 \rho R / 2\right\}
\end{array}
$$

for positive $\rho$ and $R$, where $x=\left(x^{\prime}, x^{n}\right)=\left(x^{1}, \ldots, x^{n-1}, x^{n}\right)$. We denote by $a^{i j}$, $i, j=1, \ldots, n$, the components of a positive $\mathbf{S}^{n}$ valued function on $B^{+}$satisfying

$$
\lambda|\zeta|^{2} \leq a^{i j} \zeta_{i \zeta j} \leq \Lambda|\zeta|^{2}
$$

for all $\zeta \in \mathbf{R}^{n}$ and some positive functions $\lambda, \Lambda$; and we denote by $\beta=\left(\beta^{1}, \ldots, \beta^{n}\right)$ an $\mathbf{R}^{n}$ valued function on $B^{0}$ with $\beta^{n}>0$.

LEMMA 2.1. Let $u \in C^{1}\left(B^{+} \cup B^{0}\right) \cap C^{2}\left(B^{+}\right)$satisfy the differential inequalities

$$
\begin{gathered}
L u=a^{i j} D_{i j} u \leq \lambda\left(\mu_{0}|D u|^{2}+\Phi\right) \quad \text { in } B^{+}, \\
M u=\beta^{i} D_{i} u \leq \beta^{n} \psi \quad \text { on } B^{0}
\end{gathered}
$$

for nonnegative constants $\mu_{0}, \Phi, \psi$ and suppose there are nonnegative $\mu$ and $M_{0}$ such that

$$
u \leq M_{0}, \quad \Lambda / \lambda \leq \mu \quad \text { in } B^{+}, \quad|\beta| / \beta^{n} \leq \mu \quad \text { on } B^{0} .
$$

Then there are constants $\kappa$ and $\rho$ depending only on $u$ and $\mu$ and $C$ depending also on $\mu_{0} M_{0}$ such that if $R \in\left(0, \frac{1}{4}\right)$ and $u \geq 0$ in $G(\rho, 2 R)$, then

$$
\left(\frac{1}{\left|G^{\prime}(\rho, 2 R)\right|} \int_{G^{\prime}(\rho, 2 R)} u^{\kappa}\right)^{1 / \kappa} \leq C\left(\inf _{G(\rho, R)} u+R^{2} \Phi+R \psi\right) .
$$

PROOF. Note that by considering $\tilde{u}=\left(1-e^{-\mu_{0} u}\right) / \mu_{0}$ in place of $u$, we may assume that $\mu_{0}=0$. With this assumption, we show that for $\rho=1 / 4 n \mu$, we have

$$
A=\inf _{G^{\prime}(\rho, 2 R)} u \leq 4 \inf _{G(\rho, R)} u+16(\rho R)^{2} \Phi+4 \rho R \psi .
$$

The desired result then follows by combining this inequality with the interior weak Harnack inequality

$$
\left(\frac{1}{\left|G^{\prime}(\rho, 2 R)\right|} \int_{G^{\prime}(\rho, 2 R)} u^{\kappa}\right)^{1 / \kappa} \leq C\left(\inf _{G^{\prime}(\rho, 2 R)} u+R^{2} \Phi\right),
$$

which follows from [29, Theorem 9] by means of an appropriate chaining argument. To prove (2.5), we set

$$
G_{i}^{+}=G(\rho, i R), \quad G_{i}^{0}=\partial G_{i}^{+} \cap B^{0}, \quad i=1,2,
$$

and introduce the functions

$$
\begin{aligned}
& w_{1}=4(\rho R)^{2}-\left(x^{n}\right)^{2}, \quad w_{2}=2 \rho R-x^{n} \\
& w_{3}=2-\left(x^{n}\right)^{2} / 4(\rho R)^{2}-x^{n} / 2 \rho R+\left|x^{\prime}\right|^{2} / R^{2} .
\end{aligned}
$$

Clearly $w_{i} \geq 0$ in $G_{2}^{+}$for $i=1,2,3$, and a simple calculation shows

$$
\begin{aligned}
& L w_{1} \leq-2 \lambda, \quad L w_{2} \leq 0 \quad \text { in } G_{2}^{+}, \\
& M w_{1} \leq 0, \quad M w_{2} \leq-\beta^{n} \quad \text { on } G_{2}^{0} .
\end{aligned}
$$


Moreover we have

$$
\begin{aligned}
& L w_{3} \leq \lambda\left(-\rho^{-2}+4(n-1) \mu\right) / 2 R^{2} \leq 0 \text { in } G_{2}^{+}, \\
& M w_{3} \leq \beta^{n}\left(-\rho^{-1}+8 \mu\right) / 2 R \leq 0 \text { on } G_{2}^{0},
\end{aligned}
$$

by virtue of our choice of $\rho$, and

$$
w_{3} \geq 4 \text { on } \partial G_{2}^{+} \cap\left\{\left|x^{\prime}\right|=2 R\right\}, \quad w_{3} \leq 3 \text { on } G_{1}^{+} .
$$

Thus the function $w=u+\Phi w_{1}+\psi w_{2}+A w_{3} / 4$ satisfies

$$
L w \leq 0 \quad \text { in } G_{2}^{+}, \quad M w \leq 0 \quad \text { on } G_{2}^{0}, \quad w \geq A \text { on } \partial G_{2}^{+} \backslash G_{2}^{0},
$$

so the maximum principle implies that $w \geq A$ on $G_{2}^{+}$and hence $w \geq A$ on $G_{1}^{+}$. Therefore

$$
\inf _{G(\rho, R)} u+4(\rho R)^{2} \Phi+\rho R \psi+\frac{3}{4} A \geq A
$$

which proves (2.5).

For future reference we give a simple modification of the preceding lemma.

LEMMA 2.2. Let $u \in C^{1}\left(B^{+} \cup B^{0}\right) \cap C^{2}\left(B^{+}\right)$satisfy the differential inequalities

$$
\begin{aligned}
& L u \leq \lambda\left(\mu_{0}|D u|^{2}+\Phi\left(x^{n}\right)^{\alpha-1}\right) \quad \text { in } B^{+}, \\
& M u \leq \beta^{n} \psi \text { on } B^{0}
\end{aligned}
$$

for some nonnegative constants $\mu_{0}, \Phi, \psi$ and $\alpha \in(0,1)$. Suppose (2.1) holds. Then there are constants $\kappa, \rho, C$ as in Lemma 2.1 with $C$ now depending also on $\alpha$, such that if $\kappa \in\left(0, \frac{1}{4}\right)$ and $u \geq 0$ in $G(\rho, 2 R)$, then

$$
\left(\frac{1}{\left|G^{\prime}\right|} \int_{G^{\prime}} u^{\kappa}\right)^{1 / \kappa} \leq C\left(\inf _{G(\rho, R)} u+R^{1+\alpha} \Phi+R \psi\right) \quad\left(G^{\prime}=G^{\prime}(\rho, 2 R)\right)
$$

PROOF. We proceed as in Lemma 2.1 except that here

$$
w_{1}=\left[(2 \rho R)^{1+\alpha}-\left(x^{n}\right)^{1+\alpha}\right] / \alpha(1+\alpha) .
$$

A simple consequence of Lemma 2.1 is a new Hölder estimate for solutions of linear oblique derivative problems which is the analogue of the LadyzhenskayaUral'tseva estimate [14, Chapter 10] for solutions of conormal derivative problems. For this result, $\left(a^{i j}\right)$ is a positive $\mathbf{S}^{n}$ valued function on $\Omega$ satisfying (2.1) and $\beta$ is an $\mathbf{R}^{n}$ valued function on $\partial \Omega$ such that $\beta \cdot \gamma>0$ on $\partial \Omega$.

THEOREM 2.3. Let $\partial \Omega \in C^{2}$ and let $u \in C^{1}(\bar{\Omega}) \cap C^{2}(\Omega)$ satisfy

$$
|L u| \leq \lambda\left(\mu_{0}|D u|^{2}+\Phi\right) \quad \text { in } \Omega, \quad|M u| \leq \beta \cdot \gamma \psi \quad \text { on } \partial \Omega
$$

and suppose that

$$
|u| \leq M_{0}, \quad \Lambda / \lambda \leq \mu \quad \text { in } \Omega, \quad|\beta| / \beta \cdot \gamma \leq \mu \quad \text { on } \partial \Omega .
$$

Then there are positive constants $\alpha$ depending on $\mu, \mu_{0} M_{0}, n$ and $C$ depending also on $\Phi, \psi, \Omega$ such that

$$
[u]_{\alpha ; \Omega}=\sup _{\substack{x, y \in \Omega \\ x \neq y}} \frac{|u(x)-u(y)|}{|x-y|^{\alpha}} \leq C
$$


PROOF. By virtue of [30, Corollary 11] it suffices to prove the Hölder estimate only at $\partial \Omega$, that is we must show that for all $x \in \partial \Omega$ and sufficiently close $y \in \Omega$,

$$
|u(x)-u(y)| \leq C|x-y|^{\alpha} .
$$

By means of a suitable change of variables (which increases $\mu$ by a factor of at most 2 ), we reduce to the case $\Omega=B^{+}, \partial \Omega=B^{0}$ and we may assume that $y \in G\left(\rho, \frac{1}{4}\right)$ with $\rho$ as in Lemma 2.1. For $0<R<\frac{1}{8}$ and $\rho$ as before, we set

$$
m_{R}=\inf _{G(\rho, R)} u, \quad M_{R}=\sup _{G(\rho, R)} u, \quad G(R)=G^{\prime}(\rho, R),
$$

and apply Lemma 2.1 to the functions $u-m_{4 R}$ and $M_{4 R}-u$ to obtain

$$
\begin{aligned}
& \left(\frac{1}{|G(2 R)|} \int_{G(2 R)}\left(u-m_{4 R}\right)^{\kappa}\right)^{1 / \kappa} \leq C\left(m_{R}-m_{4 R}+R^{2} \Phi+R \psi\right), \\
& \left(\frac{1}{|G(2 R)|} \int_{G(2 R)}\left(M_{4 R}-u\right)^{\kappa}\right)^{1 / \kappa} \leq C\left(M_{4 R}-M_{R}+R^{2} \Phi+R \psi\right) .
\end{aligned}
$$

Adding these inequalities yields

$$
M_{4 R}-m_{4 R} \leq C\left(M_{4 R}-m_{4 R}+m_{R}-M_{R}+R^{2}+R\right)
$$

from which the estimate follows by a standard argument (see [9, Theorem 8.22]).

We remark that Lemma 2.2 can be used to relax the regularity hypothesis on $\Omega$ to $\partial \Omega \in C^{1, \delta}$ for some $\delta \in(0,1)$. Also we could have employed the full Harnack inequality [29, Corollary 10], instead of the weak Harnack inequality (2.6), to derive the Hölder estimate (2.11). However this will not be possible when we come to the Hölder estimates of derivatives.

The Hölder estimate for solutions of the boundary value problem (1.1), (1.2) is now a simple consequence of Theorem 2.3.

THEOREM 2.4. Let $\partial \Omega \in C^{2}$ and let $u \in C^{1}(\bar{\Omega}) \cap C^{2}(\Omega)$ be a solution of (1.1),(1.2) with $|u| \leq M_{0}$ in $\Omega$ and F1, F2, G2 holding. Then there are constants $\alpha=\alpha\left(n, M_{0}, \mu, \mu_{0}\right)>0$ and $C=C\left(n, M_{0}, \mu, \mu_{0}, \Omega\right)$ such that

$$
[u]_{\alpha ; \Omega} \leq C \text {. }
$$

PROOF. Using the mean value theorem, we can write (1.1) in the form

$$
F^{i j}(x, u, D u, s) D_{i j} u+F(x, u, D u, 0)=0 \text { in } \Omega
$$

for some $s=s(x) \in \mathbf{S}^{n}$, and (1.2) in the form

$$
\left(G_{p}(x, u, q) \cdot \gamma\right)(D u \cdot \gamma)+G\left(x, u, D^{\prime} u\right)=0 \text { on } \partial \Omega
$$

for some $q=q(x) \in \mathbf{R}^{n}$ with $D^{\prime} u=D u-(D u \cdot \gamma) \gamma=q-(q \cdot \gamma) \gamma$. Thus the hypotheses of Theorem 2.3 are fulfilled with

$$
a^{i j}=F^{i j}(x, u, D u, s), \quad \beta^{i}=\left[\gamma^{i}+\beta^{*} D_{i}^{\prime} u /\left|D^{\prime} u\right|\right] G_{p}(x, u, q)
$$

for some $\beta^{*}(x, u, D u)$ satisfying $\left|\beta^{*}\right| \leq \mu_{0}$.

It is interesting to compare Theorem 2.3 and its proof with Krylov's boundary Hölder estimate [12, Theorem 4.1] (as simplified by Caffarelli). Indeed if we assume, 
instead of (2.3), the Dirichlet boundary condition $u=0$ on $B^{0}$ in Lemma 2.1 , then using the replacement functions

$$
w_{1}=2 \rho R x^{n}-\left(x^{n}\right)^{2}, \quad w_{2}=0, \quad w_{3}=x_{n}\left(1-x_{n} / 2 \rho R+\left|x^{\prime}\right|^{2} / R^{2}\right),
$$

and $\rho=1 / 8 n \mu$, we obtain a weak Harnack inequality for the ratio $v=u / x^{n}$, namely

$$
\left(\frac{1}{\left|G^{\prime}\right|} \int_{G^{\prime}} v^{\kappa}\right)^{1 / \kappa} \leq C\left(\inf _{G(\rho, R)} v+R \Phi\right) \quad\left(G^{\prime}=G^{\prime}(\rho, 2 R)\right) .
$$

As before we need only apply the interior weak Harnack inequality (2.6) to the function $u$, observing that in $G^{\prime}(\rho, 2 R)$ we have $u / 3 \rho R \leq v \leq u / 2 \rho R$. Accordingly, by the proof of Theorem 2.3 (and the boundary Lipschitz estimate [9, Theorem 14.1], for any function $u \in C^{0}\left(B^{+} \cup B^{0}\right) \cap C^{2}\left(B^{+}\right)$satisfying the differential inequality (2.10) in $B^{+}$together with $u=0$ on $B^{0},|u| \leq M_{0}$, we obtain the Hölder estimate

$$
\operatorname{osc}_{B_{R}^{+}} v \leq C\left(M_{0}+\Phi\right) R^{\alpha}
$$

where $\alpha$ and $C$ are positive constants depending on $n, \mu$, and $\mu_{0} M$. We shall use estimate (2.14) in $\S 6$. An extension, along similar lines to Lemma 2.2, is given in [22].

To conclude this section we remark that conditions F2 and G2, which are used in Theorem 2.4 and elsewhere in this paper to write the problem (1.1), (1.2) in a quasilinear form, may be replaced by more general conditions. When $\lambda$ and $\chi$ are bounded away from zero, they follow naturally from F1, F3, and G3. Otherwise we may, for example, replace them by the conditions

$$
F(x, z, p, \bar{r})=0, \quad G\left(x, z, p^{\prime}, \bar{p}_{n}\right)=0
$$

for some $\bar{r}=\bar{r}(x, z, p), \bar{p}_{n}=p_{n}\left(x, z, p^{\prime}\right)$ satisfying

$$
|\bar{r}| \leq \mu_{0}(|z|)\left(1+|p|^{2}\right), \quad\left|\bar{p}_{n}\right| \leq \mu_{0}(|z|)\left(1+\left|p^{\prime}\right|\right)
$$

(see [17, Lemma 2.1; 18, Lemma 4.2]).

3. Gradient bounds. In this section we prove various gradient bounds for solutions of the boundary value problem (1.1), (1.2) under hypotheses F1, F2, F3, G2, G3 as well as other closely related hypotheses. The method of proof combines ingredients from the authors' previous works [18 and 30], although for computational reasons we employ a construction analogous to that in [2 and 4] (instead of the change of dependent variable in [30]) and locally flatten the boundary. The main new technical difficulty in the present situation arises from cubic terms in the gradient, introduced through differentiation of the boundary condition (1.2).

Throughout this section we shall abbreviate $F^{i}=F_{p_{i}}, G^{i}=G_{p_{i}}$ and use the differential operators $\delta=D_{z}+|p|^{-2} p \cdot D_{x}, \bar{\delta}=p \cdot D_{p}$. We begin with a local gradient estimate to illustrate the general argument. This estimate was proved in a slightly different form in [30] via a change of dependent variable (see also [2]).

LEMMA 3.1. Let $u \in C^{3}(\Omega) \cap C^{1}(\bar{\Omega})$ be a solution of $F[u]=0$ in $\Omega$ and suppose there are nonnegative constants $\mu, \mu_{1}, M$ such that for any $\varepsilon \in(0,1)$,

$$
\Lambda|p|^{2} \leq \mu \mathcal{E}, \quad|p|\left|F_{p}\right|, \delta F \leq \frac{\mu_{1}}{\varepsilon} \mathcal{E}+\varepsilon \mathcal{E}_{2} /|p|^{2}
$$


for $|p| \geq M$. Then there are constants $C$ and $\theta$ depending only on $n, \mu$, and $\mu_{1}$ such that if $B=B_{R}(y)$ is any ball in $\Omega$ and $\alpha=\operatorname{osc}_{B} u \leq \theta$, then

$$
|D u(y)| \leq C \alpha / R+M \text {. }
$$

Further the estimate (3.2) remains valid if $B$ is replaced by $B \cap \Omega$ for any ball $B=B_{R}(y)$ with $y \in \Omega$ and $|D u| \leq M$ on $\partial \Omega \cap B$.

PROOF. Without loss of generality we can take $y=0$, and assume $\alpha<\frac{1}{2} \mu_{1}$ and $\varepsilon=2 \alpha \mu_{1}$ in (3.1). For $\beta \in\left(0, \frac{1}{2}\right)$ set

$$
\begin{gathered}
\eta=\left(1-|x|^{2} / R^{2}\right)^{2}, \quad M_{1}=\sup _{B_{R}} \eta|D u|^{2}, \quad M_{0}=\sup _{B_{R}} u \\
u^{*}=\exp \left(u-M_{0}\right) / \alpha, \quad w=\eta|D u|^{2}+\beta M_{1} u^{*} .
\end{gathered}
$$

As in $[30,(4.7)]$, we apply the operator $D_{k} u D_{k}$ to the equation $F[u]=0$ to obtain

$$
\begin{aligned}
F^{i j} D_{i j} w+B^{i} D_{i} w= & |D u|^{2}\left\{F^{i j} D_{i j} \eta-(2 / \eta) F^{i j} D_{i} \eta D_{j} \eta-F^{i} D_{i} \eta-\eta \delta F\right\} \\
& +(\beta / \alpha) M_{1} u^{*}\left\{F^{i} D_{i} u-(2 / \eta) F^{i j} D_{i} \eta D_{j} u+F^{i j} D_{i j} u\right\} \\
& +2 \eta \mathcal{E}_{2}+\beta \alpha^{-2} M_{1} u^{*} \mathcal{E},
\end{aligned}
$$

where $B^{i}=F^{i}-(2 / \eta) F^{i j} D_{j} \eta$. Moreover a simple calculation gives

$$
|D \eta| \leq 4 \eta^{1 / 2} / R, \quad F^{i j} D_{i j} \eta \geq-4 F^{i i} / R^{2} \geq-4 n \Lambda R^{2} .
$$

From these estimates and the structure conditions (3.1), it follows that, on the set where $|D u| \geq M$,

$$
\begin{aligned}
& F^{i j} D_{i j} w+B^{i} D_{i} w \\
& \geq \mathcal{E}\left\{-4 n \mu R^{-2}-32 n \mu R^{-2}-2 \eta^{1 / 2}|D u| / \alpha R-\eta|D u|^{2} /(2 \alpha)\right. \\
& -\beta M_{1} u^{*} / 2 \alpha^{2}-8 \beta M_{1} u^{*} \mu /\left(\alpha \eta^{1 / 2}|D u| R\right) \\
& \left.-\beta^{2} M_{1}^{2} u^{*} n \mu /\left(2 \alpha^{2} \eta|D u|^{2}\right)+\beta M_{1} u^{*} / \alpha^{2}\right\} \\
& +\mathcal{E}_{2}\left\{-8 \mu_{1} \alpha \eta^{1 / 2} /(R|D u|)-2 \mu_{1} \alpha \eta-2 \mu_{1} M_{1} u^{*} \beta /|D u|^{2}+3 \eta / 2\right\} \text {. }
\end{aligned}
$$

Now we use the inequalities $\beta \leq \frac{1}{2}$ and $u^{*} \leq 1$ to infer that $M_{1} \leq w \leq \eta|D u|^{2}+$ $M_{1} / 2$ at a point $x_{0}$ where $w$ attains its maximum, that is, $\eta|D u|^{2} \geq M_{1} / 2$. Hence at $x_{0}$,

$$
\begin{aligned}
F^{i j} D_{i j} w \geq & \mathcal{E}\left\{-(4 n+32) \mu / R^{2}-2 M_{1}^{1 / 2} /(\alpha R)-M_{1} / \alpha\right\} \\
& +\left(u^{*} \beta / \alpha^{2}\right) \mathcal{E}\left\{-8 \mu \alpha M_{1}^{1 / 2} / R-\beta n \mu M_{1}+M_{1} / 2\right\} \\
& +\eta \mathcal{E}_{2}\left\{-8 \mu_{1} \alpha /\left(R M_{1}^{1 / 2}\right)-2 \alpha \mu_{1}-4 \beta \mu_{1}+3 / 2\right\} .
\end{aligned}
$$

Clearly the coefficient of $\mathcal{E}_{2}$ will be positive if

$$
\left(16 \mu_{1} \alpha / R\right)^{2}<M_{1}, \quad \alpha \leq 1 / 4 \mu_{1}, \beta \leq 1 / 8 \mu_{1},
$$

and the coefficient of $\left(u^{*} \beta / \alpha^{2}\right) \mathcal{E}$ will be positive if $(16 \mu \alpha / R)^{2}<M_{1}, \beta \leq 1 / 8 n \mu$. Hence for $\alpha \leq 1 / 4 \mu_{1}, \beta=\min \left\{1 / 8 \mu_{1}, 1 / 8 n \mu\right\}$, and $M_{1}>16\left(\mu_{1}+\mu\right)(\alpha / R)^{2}$, we have, since $u^{*} \geq e^{-1}>1 / 3$,

$$
F^{i j} D_{i j} w \geq \mathcal{E}\left\{\beta M_{1} / 24 \alpha^{2}-(4 n+32) \mu / R^{2}-2 M_{1}^{1 / 2} /(\alpha R)-M_{1} / \alpha\right\} .
$$


Therefore we have $F^{i j} D_{i j} w>0$ at $x_{0}$ if

$$
\alpha \leq \theta=1 /\left[C(n)\left(\mu+\mu_{1}\right)\right]
$$

and

$$
M_{1}>(C \alpha / R)^{2}, \quad C=C(n)\left(\mu+\mu_{1}\right) .
$$

Since $F^{i j} D_{i j} w \leq 0$ at a maximum of $w$, it follows that

$$
M_{1} \leq \max \left\{(C \alpha / R)^{2}, M^{2}\right\} \text { for } \alpha \leq \theta,
$$

where $C$ and $\theta$ are given by (3.3).

Let us note that (3.1) follows from the easily verified conditions

$$
\Lambda|p|^{2} \leq \mu \mathcal{E}, \quad|p|\left|F_{p}\right|, \delta F \leq \mu_{1}\left(\mathcal{E}+\left(\Lambda \mathcal{E}_{2}\right)^{1 / 2}\right)
$$

and that $(3.1)^{\prime}$ follows from F1, F3.

Futher, Lemma 3.1 continues to hold for $u \in W^{2, q}(\Omega)$ when $q>2 n(q>n$ if $(3.1)^{\prime}$ is valid). In this case we replace the differentiation of $F[u]=0$ by a differencing. Setting

$$
\Delta_{t}^{(h)} u(x)=\left[u\left(x+h e_{t}\right)-u(x)\right] / h,
$$

where $e_{t}$ is the standard $t$ th unit vector in $\mathbf{R}^{n}, h>0$, we obtain for

$$
w_{h}=\eta \sum\left(\Delta_{t}^{(h)} u\right)^{2}+\beta M_{1, h} u^{*}
$$

the differential inequality

$$
F^{i j} D_{i j} w_{h}+B^{i} D_{i} w_{h} \geq-\varepsilon(h) w_{h}, \quad \text { on } B_{R, h}=\left\{x \in B_{R} \mid w_{h} \geq \frac{3}{4} \sup _{B_{R}} w_{h}\right\},
$$

where $\|\varepsilon(h)\|_{L^{n}\left(B_{R, h}\right)} \rightarrow 0$ as $h \rightarrow 0$, provided $M_{1, h} \geq \max \left\{(C \alpha / R)^{2}, M^{2}\right\}, \alpha \leq \theta$, with $C$ and $\theta$ given by (3.3). Since $\left\|B^{i}\right\|_{n}$ is bounded uniformly with respect to $h$, the Aleksandrov maximum principle [9, Chapter 9] provides the desired estimate for $w_{h}$ if $h$ is small enough.

Our next step is to bound the tangential derivatives of $u$ near a flat boundary position. To this end we set

$$
\begin{aligned}
& p^{\prime}=\left(p_{1}, \ldots, p_{n-1}\right), \quad D^{\prime}=\left(D_{1}, \ldots, D_{n-1}\right), \\
& C^{2}=F^{i j} r_{i k} r_{j k}, \quad \delta_{T}=D_{z}+\left|p^{\prime}\right|^{2} p^{\prime} \cdot D_{x},
\end{aligned}
$$

where here and in the following lemma, we adopt the convention that the index $k$ only goes from 1 to $n-1$. We also recall the definitions of $B^{+}$and $B^{0}$ from $\S 2$.

LEMMA 3.2. Let $u \in C^{2}\left(B^{+} \cup B^{0}\right) \cap C^{3}\left(B^{+}\right)$be a solution of

$$
F[u]=0 \quad \text { in } B^{+}, \quad G[u]=0 \text { on } B^{0} .
$$

Suppose that there are nonnegative constants $\mu, \mu_{1}, \mu_{2}, \bar{\mu}_{2}, M$ such that

$$
\begin{gathered}
|p|\left|G_{p}\right|, \delta_{T} G \leq \mu_{1}|p| \chi \\
\Lambda|p|^{2} \leq \mu \mathcal{E} \\
\Lambda|r|,|p|\left|F_{p}\right|, \delta F, \delta_{T} F \leq \mu_{2} \mathcal{E}+\bar{\mu}_{2} C^{2} /\left|p^{\prime}\right|^{2} \quad \text { if } F(X)=0,
\end{gathered}
$$


whenever $\left|p^{\prime}\right| \geq M$. Suppose also that there is a continuous increasing function $\omega$ with $\omega(0)=0$ such that

$$
|u(x)-u(0)| \leq \omega(|x|), \quad|D u(x)| \leq \omega(|x|) / x^{n} \quad \text { for all } x \in B^{+} .
$$

If also $\bar{\mu}_{2}$ is sufficiently small (depending only on $\mu_{1}$ ), then

$$
\left|D^{\prime} u(0)\right| \leq C\left(n, \mu, \mu_{1}, \mu_{2}, \bar{\mu}_{2}, M, \omega\right) .
$$

Before proving this lemma, we discuss condition (3.7) which is only required to hold when $F(X)=0$. Unlike the proof of the interior gradient bound, the present proof uses a control of $D_{n n} u$ in terms of $D_{i j} u$ (for $i+j<2 n$ ), which can only be obtained via the equation. For our proof, condition (3.7) is the correct form of this control. We note here that F1 and F3 imply that the quantities in the left-hand side of (3.7) are bounded by $\lambda C\left(n, \mu, \mu_{1}\right)\left(|r|+|p|^{2}+1\right)$ while F1 and F2 imply that

$$
\left|r_{n n}\right| \leq C(n, \mu)\left(\sum_{i+j<2 n}\left(r_{i j}\right)^{2}\right)^{1 / 2}+\mu_{0}\left(1+|p|^{2}\right) \quad \text { if } F(X)=0
$$

and hence, where $|p|>1$,

$$
\left|r_{n n}\right| \leq \frac{1}{\lambda} C\left(n, \mu, \mu_{0}\right)\left(\left[\mathcal{E} C^{2} /|p|\right]^{1 / 2}+\mathcal{E}\right) .
$$

Therefore (3.7) holds in the uniformly elliptic case for any $\bar{\mu}_{2}$ with

$$
\mu_{2}=C\left(n, \mu, \mu_{0}, \mu_{1}\right)\left(1+1 / \bar{\mu}_{2}\right) \text {. }
$$

ProOF of Lemma 3.2. Let $R \in(0,1)$ be a constant at our disposal and set

$$
\begin{aligned}
& B_{R}^{+}=\left\{x \in B^{+}|| x \mid<R\right\}, \quad B_{R}^{0}=\left\{x \in B^{0}|| x \mid<R\right\}, \\
& M_{0}=\sup _{B_{R}^{+}} u, \quad w^{\prime}=\left|D^{\prime} u\right|^{2}, \quad M_{1}=\sup _{B_{R}^{+}} \eta w^{\prime},
\end{aligned}
$$

where $\eta$ is as in Lemma 3.1. With $\alpha_{1}>4$ and $\alpha_{2}>0$ constants to be chosen, set

$$
u^{*}=\exp \alpha_{1}\left(u-M_{0}\right), \quad v=\left(1+|D u|^{2}\right)^{1 / 2}, \quad w=\eta w^{\prime}+M_{1} u^{*} / \alpha_{1}+\alpha_{2} M_{1} v x^{n} .
$$

We then determine $R$ from $\alpha_{1}, \alpha_{2}, M$, and $\omega$ so that $R \leq 2 / M$ and

$$
\alpha_{1}\left(M_{0}-u\right) \leq 1, \quad \alpha_{2} v x^{n} \leq 1 / 4 \quad \text { in } B_{R}^{+} .
$$

If $M_{1} \leq R^{-2}$, then the estimate on $w^{\prime}(0)$ is clear once we have determined $\alpha_{1}$ and $\alpha_{2}$. We now determine suitable $\alpha_{1}$ and $\alpha_{2}$ so that $M_{1} \leq R^{-2}$. Suppose now that $M_{1}>R^{-2}$ and let $x_{0}$ be a point in $\overline{B_{R}^{+}}$where $w$ attains its maximum. As before $\eta w^{\prime}\left(x_{0}\right) \geq M_{1} / 2$ and, in particular, $\eta\left(x_{0}\right) \neq 0$.

If $x_{0}^{n}=0$, then by applying the operator $D_{k} u D_{k}$ to the equation $G[u]=0$, we obtain $G^{i} D_{i} w^{\prime}=-2\left(\delta_{T} G\right) w^{\prime}$. Therefore for $w^{\prime}>M^{2}$, we have

$$
\begin{aligned}
G^{i} D_{i} w & =\left(G^{i} D_{i} \eta\right) w^{\prime}-2 \eta\left(\delta_{T} G\right) w^{\prime}+M_{1} u^{*} \bar{\delta} G+\alpha_{2} M_{1} v \chi \\
& >\chi\left[-\mu_{1} w^{\prime}|D \eta|-2 \mu_{1} \eta w^{\prime}|D u|-\mu_{1} M_{1}|D u|+\alpha_{2} M_{1}|D u|\right] \\
& \geq \chi\left[-4 \mu_{1} \eta^{1 / 2} w^{\prime} / R-3 \mu_{1} M_{1}|D u|+\alpha_{2} M_{1}|D u|\right] \\
& \geq \chi M_{1}|D u|\left(-7 \mu_{1}+\alpha_{2}\right) .
\end{aligned}
$$


Hence $G^{i} D_{i} w>0$ at $x_{0}$ if $\alpha_{2}=7 \mu_{1}$ because $w^{\prime}\left(x_{0}\right) \geq M_{1} / 2>M^{2}$. Therefore $w$ cannot have a maximum on $B_{R}^{0}$ if $M_{1}>R^{-2}$ and $\alpha_{2}=7 \mu_{1}$.

If $x_{0}^{n}>0$, then a direct calculation yields

$$
\begin{aligned}
D_{i} w= & \eta D_{i} w^{\prime}+w^{\prime} D_{i} \eta+M_{1} u^{*} D_{i} u+\alpha_{2} M_{1}\left(x^{n} D_{i j} u \nu^{j}+v \delta^{i n}\right) \\
D_{i j} w= & w^{\prime} D_{i j} \eta+D_{i} \eta D_{j} w^{\prime}+D_{i} w^{\prime} D_{j} \eta+2 \eta D_{i j k} u D_{k} u+2 \eta D_{i k} i D_{j k} u \\
& +\alpha_{1} M_{1} u^{*} D_{i} u D_{j} u+M_{1} u^{*} D_{i j} u \\
& +\alpha_{2} M_{1}\left(x^{n} D_{i j m} u \nu^{m}+x^{n} D_{i m} u g^{m s} D_{j s} u+\delta^{i n} D_{j m} u \nu^{m}+D_{i m} u \nu^{m} \delta^{j n}\right),
\end{aligned}
$$

where $\nu=D u / v$ and $g^{m s}=\left(\delta^{m s}-\nu^{m} \nu^{s}\right) / v$. Moreover, differentiating $F[u]=0$ with respect to $x^{m}$ gives

$$
F^{i j} D_{i j m} u=-F^{i} D_{i m} u-F_{z} D_{m} u-F_{m}
$$

so, with $B^{i}$ as in the proof of Lemma 3.1, we have

$$
\begin{aligned}
F^{i j} D_{i j} w+B^{i} D_{i} w=\alpha_{1} M_{1} u^{*} \mathcal{E}+2 \eta C^{2}+\alpha_{2} M_{1} x^{n} F^{i j} D_{i m} u g^{m s} D_{j s} u \\
\quad+\left(F^{i j} D_{i j} \eta-(2 / \eta) F^{i j} D_{i} \eta D_{j} \eta\right) w^{\prime}-(2 / \eta) M_{1}\left(u^{*} F^{i j} D_{i} \eta D_{j} u+\alpha_{2} v F^{i n} D_{i} \eta\right) \\
\quad+M_{1}\left((2 / \eta) \alpha_{2} x^{n} F^{i j} D_{i} \eta D_{j m} u \nu^{m}-2 \alpha_{2} F^{i n} D_{i m} u \nu^{m}+u^{*} F^{i j} D_{i j} u\right) \\
\quad+M_{1}\left(\alpha_{2} v F^{n}+u^{*} \bar{\delta} F-\alpha_{2} v x^{n}|\nu|^{2} \delta F\right)+\left(F^{i} D_{i} \eta-2 \eta \delta_{T} F\right) w_{T} .
\end{aligned}
$$

We now proceed as in Lemma 3.1, using conditions (5.3) and the inequalities $|\nu| \leq 1$, $\left(g^{i j}\right) \geq 0$ to obtain

$$
F^{i j} D_{i j} w+B^{i} D_{i} w \geq\left(\frac{1}{3} \alpha_{1}-c\left(n, \mu, \mu_{2}\right)\right) M_{1} \mathcal{E}+\left(2-c\left(\alpha_{2}\right) \bar{\mu}_{2}\right) \eta C^{2} .
$$

Recalling that $\alpha_{2}=7 \mu_{1}$, we see that the right-hand side of this inequality will be positive if $\bar{\mu}_{2}$ is sufficiently small (depending only on $\mu_{1}$ ) and $\alpha_{1}$ is sufficiently large. With these choices for $\alpha_{1}$ and $\alpha_{2}, w$ cannot have a maximum where $x^{n}>0$ and therefore $M_{1} \leq R^{-2}$.

As before, Lemma 3.2 is also valid for $W^{2, q}(q>2 n)$ solutions of (3.4), and (3.7) will usually be inferred from

$$
\Lambda|r|,|p|\left|F_{p}\right|, \delta F, \delta_{T} F \leq \mu_{1}\left(\mathcal{E}+\left(\Lambda C^{2}\right)^{1 / 2}\right) \quad \text { if } F(X)=0 .
$$

We also note that our estimate on $\left|D^{\prime} u\right|$ was achieved without first estimating the ratio $|D u| /\left|D^{\prime} u\right|$, as was done in [18].

Now we combine the preceding lemmata to bound the full gradient of solutions of (1.1), (1.2) in arbitrary smooth domains.

THEOREM 3.3. Let $\partial \Omega \in C^{3}$ and let $u \in C^{2}(\bar{\Omega}) \cap C^{3}(\Omega)$ be a solution of (1.1), (1.2) with $|u| \leq M$ in $\Omega$ and suppose that $F$ and $G$ satisfy conditions F1, F2, F3, G2 and G3. Then

$$
\sup _{\Omega}|D u| \leq C\left(n, M, \mu, \mu_{0}, \mu_{1}, \Omega\right) .
$$

ProOF. First we estimate $|D u|$ on $\partial \Omega$. To this end we consider a point on $\partial \Omega$, which we may take to be the origin, where $|D u|$ attains its maximum over $\partial \Omega$. Since the form of our hypotheses is unchanged under any $C^{3}$ change of coordinates (although the values of the constants will change), we may also assume that $\Omega \cap B_{1}=$ $B^{+}$and $\partial \Omega \cap B_{1}=B^{0}$. It then follows from G2 that

$$
\left|D_{n} u(0)\right| \leq \mu_{0}\left(1+\left|D^{\prime} u(0)\right|\right)
$$


and hence we need only estimate $\left|D^{\prime} u(0)\right|$. This estimate is immediate from Lemma 3.2 since (3.5) follows from G3, (3.6) follows from F1, (3.7) follows from F1, F2, and F3, and (3.9) follows from Theorem 2.3 and Lemma 3.1.

This estimate for $|D u|$ on $\partial \Omega$, and another application of Lemma 3.1 give the full estimate.

Again we can relax the regularity of $u$ to $W^{2, q}(q>n)$. Also the conditions on $F_{z}$ and $F_{x}$ can be relaxed to a one-sided estimate on a suitable linear combination of these derivatives corresponding to $\delta F$ and $\delta_{T} F$ in the transformed domain. We shall discuss presently a version of this one-sided bound without performing a change of coordinates. The gradient estimate near $\partial \Omega$ may also be effected without a direct use of the interior estimate, Lemma 3.1, although such an approach seems more complicated.

So far our gradient estimates have all been local in character. This localness was used crucially to make certain terms small by virtue of our modulus of continuity estimates. We now consider an alternative approach which avoids the modulus of continuity estimates by strengthening certain of our structure conditions. This alternative approach has several other attractive features. It allows us to obtain global estimates directly under the strengthened structure conditions and it does not use a flattening of the boundary. In this last respect, it can be used to give an explicit version of the one-sided estimates on combinations of $F_{z}$ and $F_{x}$ which were used in the proof of Theorem 3.3. Also we are able to obtain gradient estimates which are independent of the ratio $\left|G_{p}\right| / \chi$; the utility of this will be shown in an example.

For our alternative approach, we define

$$
\Sigma=\{x \in \Omega: d(x)=\operatorname{dist}(x, \partial \Omega)<\tau\},
$$

where $\tau>0$ is so small that $d \in C^{3}(\bar{\Sigma})$, which is always possible if $\partial \Omega \in C^{3}$. We then set $D d=\gamma$ in $\Sigma$ and note that

$$
|\gamma|=1, \quad \gamma^{i} D_{i} \gamma^{j}=\gamma^{i} D_{j} \gamma^{i}=0 \quad \text { in } \Sigma
$$

and that this $\gamma$ is a $C^{2}$ extension of the normal field on $\partial \Omega$ into $\Sigma$. We also define

$$
\begin{aligned}
& c^{i j}=\delta^{i j}-\gamma^{i} \gamma^{j}, \quad D_{i}^{\prime}=c^{i j} D_{j}, \quad D^{\prime}=\left(D_{1}^{\prime}, \ldots, D_{n}^{\prime}\right), \quad p_{i}^{\prime}=c^{i j} p_{j}, \\
& \delta^{\prime}=D_{z}+\left|p^{\prime}\right|^{-2} p^{\prime} \cdot D x-\frac{1}{2}\left|p^{\prime}\right|^{-2} p_{i} p_{j} D\left(c^{i j}\right) \cdot D_{p} .
\end{aligned}
$$

Note that $\delta^{\prime}=\delta_{T}$ near a flat boundary portion. Further discussion of $\delta^{\prime}$ can be found in $[18, \S 4]$ (where there is a minus sign missing in the definition of $c_{i}$ on $\mathrm{p}$. $59)$.

THEOREM 3.4. Let $\partial \Omega \in C^{3}$ and let $u \in C^{2}(\bar{\Omega}) \cap C^{3}(\Omega)$ be a solution of (1.1), (1.2) with

$$
(1+|D u|) d \leq K, \quad|u| \leq M_{0} \quad \text { in } \Sigma .
$$

Suppose $F$ and $G$ satisfy the conditions

$$
\Lambda|r|, \delta F,|p|\left|F_{p}\right| \leq \mu_{1} \mathcal{E}+\bar{\mu}_{1} C^{2} /\left|p^{\prime}\right|^{2}, \quad \delta^{\prime} F \leq \bar{\mu}|p| \mathcal{E}+\bar{\mu}_{1} C^{2} /\left|p^{\prime}\right|^{2}
$$

for $F(X)=0,\left|p^{\prime}\right|>M, x \in \Sigma$, and

$$
-\bar{\delta} G, \delta^{\prime} G \leq \bar{\mu}(|p|)|p| \chi
$$


for $\left|p^{\prime}\right|>M$, where $\bar{\mu}$ is a decreasing function with $\lim _{t \rightarrow \infty} \bar{\mu}(t)=0$. If $\bar{\mu}_{1}$ is sufficiently small, then

$$
\sup _{\Sigma}\left|D^{\prime} u\right| \leq C\left(K, M, M_{0}, \bar{\mu}, \mu_{1}, \bar{\mu}_{1}, \mu\right)
$$

PROOF. Let $\alpha_{1} \geq 4$ and $\alpha_{2}<1 / 4 K$ be constants at our disposal and set

$$
\begin{gathered}
w^{\prime}=\left|D^{\prime} u\right|^{2}=c^{i j} D_{i} u D_{j} u, \quad M_{1}=\sup _{\varepsilon} w^{\prime}, \quad u^{*}=\exp \alpha_{1}\left(u-M_{0}\right) \\
w=w^{\prime}+M_{1} u^{*} / \alpha_{1}+\alpha_{2} M_{1} v d
\end{gathered}
$$

where $v=\left(1+|D u|^{2}\right)^{1 / 2}$, as in Lemma 3.2. If $M_{1} \leq 4(K / \tau)^{2}+2 M^{2}$, we are done. Thus we may assume that $M_{1}>4(K / \tau)^{2}+2 M^{2}$ which implies that $w$ cannot achieve its maximum over $\bar{\Sigma}$ where $d=\tau$. Thus $w \geq M_{1} / 2$ and $\left|D^{\prime} u\right| \geq M$ at a maximum point $x_{0}$. If $x_{0} \in \partial \Omega$, then

$$
G^{i} D_{i} w\left(x_{0}\right) \geq M_{1} v \chi\left(\alpha_{2}-2 \bar{\mu}\left(\left|D^{\prime} u\left(x_{0}\right)\right|\right)\right),
$$

so $w$ cannot have a maximum on $\partial \Omega$ if $M_{1}>\left(\bar{\mu}^{-1}\left(\alpha_{2} / 2\right)\right)^{2}$.

If $x_{0} \in \Sigma$, we obtain an expression for $F^{i j} D_{i j} w+F^{i} D_{i} w$, all of whose terms can be estimated as before with two exceptions:

$$
\begin{aligned}
4 F^{i j} D_{i k} u D_{j}\left(c^{k m}\right) D_{m} u & =-4 F^{i j} D_{i k} u D_{j} u D_{j} \gamma^{k} D u \cdot \gamma-4 F^{i j} D_{i k} u \gamma^{k} D_{j} \gamma^{m} D_{m} u \\
& \geq-C(\Omega) \mu\left(\varepsilon C^{2}\right)^{1 / 2}-C(\Omega) w_{T}^{1 / 2} \Lambda \mid D^{2} u
\end{aligned}
$$

and

$$
2 F^{i j} D_{i j}\left(c^{k m}\right) D_{k} u D_{m} u \geq-C(\Omega) \mu \mathcal{E} .
$$

Hence, if $\alpha_{2} \leq 1$ and $\bar{\mu}=\bar{\mu}\left(M_{1} / 2\right) \leq 1$, then

$F^{i j} D_{i j} w+F^{i} D_{i} w \geq\left\{-C\left(\Omega, \mu, \mu_{1}, n\right)\left(\alpha_{2}+\bar{\mu}+u^{*}\right)+\alpha_{1} u^{*}\right\} M_{1} \mathcal{E}+\left\{2-c\left(\alpha_{2}\right) \bar{\mu}_{1}\right\} C^{2}$.

By first choosing $\alpha_{1}$ sufficiently large, then $\alpha_{2} \leq u^{*}$ and then $\bar{\mu}_{1}$ small, we see that $w$ cannot reach its maximum in $\Sigma$ if $\bar{\mu}\left(M_{1} / 2\right) \leq u^{*}$. As before this leads to the desired estimate.

We remark that, by virtue of the proof of Lemma 3.1, the form of the interior gradient estimate given in (3.11) is a consequence of a slight strengthening of (3.12), namely,

$$
|p|\left|F_{p}\right| \leq \mu_{1} \mathcal{E}+\bar{\mu}_{1} \varepsilon_{2} /|p|^{2}, \quad \delta F \leq \bar{\mu}(|p|) \mathcal{E}+\bar{\mu}_{1} \varepsilon_{2} /|p|^{2} .
$$

Moreover we can take $\bar{\mu}$ in (3.12) and (3.13) (but not (3.12)) to be a constant if we have a modulus of continuity estimate for $u$. By using the ideas of the proof of Theorem 3.4, we can prove Theorem 3.3 without flattening the boundary, in which case F3 and G3 can be relaxed to

$$
\begin{aligned}
& |p|\left|F_{p}\right|, \delta F, \delta^{\prime} F \leq \mu_{1} \lambda\left(1+|p|^{2}+|r|\right), \\
& |p|\left|G_{p}\right|, \delta^{\prime} G \leq \mu_{1} \chi(1+|p|) .
\end{aligned}
$$

Also, we can replace $-\bar{\delta} G$ by $\bar{\delta} G$ in (3.12) by a simple modification of the proof, and if $\bar{\mu}(t)=O(1 / t)$ in (3.13) (but not necessarily in (3.12)), then any interior gradient estimate can be substituted in (3.11). In this case, we take $w=w^{\prime}+M_{1} u^{*} / \alpha_{1}+$ $\alpha_{2} M_{1} d$ with $\alpha_{2}$ a sufficiently large constant and $\tau \leq 1 / 4 \alpha_{2}$. 
As an application of Theorem 3.4, we consider the contact angle boundary condition (1.4) with constant contact angle, i.e.,

$$
G[u]=\gamma \cdot D u-\phi\left(1+|D u|^{2}\right)^{1 / 2}=0 \text { on } \partial \Omega
$$

for some constant $\phi \in(-1,1)$. By direct calculation, we have

$$
\begin{aligned}
& G_{p}=\gamma-\phi\left(1+|p|^{2}\right)^{1 / 2} p, \quad G_{z}=0, \quad G_{x}=\left(D \gamma^{j}\right) p_{j}, \\
& \chi=1-\phi p \cdot \gamma /\left(1+|p|^{2}\right)^{1 / 2}, \quad \bar{\delta} G=p \cdot \gamma-\phi|p|^{2} /\left(1+|p|^{2}\right)^{1 / 2}, \\
& \delta^{\prime} G=D_{i} \gamma^{j} p_{i} p_{j} /\left|p^{\prime}\right|^{2}-\left(\gamma-\phi\left(1+|p|^{2}\right)^{-1 / 2} p\right) \cdot D\left(c^{i j}\right) p_{i} p_{j} / 2\left|p^{\prime}\right|^{2} \\
& \quad=\chi D_{i} \gamma^{j} p_{i} p_{j} /\left|p^{\prime}\right|^{2} .
\end{aligned}
$$

It is readily checked that

$$
D_{i} \gamma^{j} p_{i} p_{j} \leq C(\Omega)\left|p^{\prime}\right|^{2} \quad \text { in } \Sigma
$$

and that

$$
\bar{\delta} G[u]=\phi\left(1+|D u|^{2}\right)^{-1 / 2} \quad \text { if } G[u]=0 .
$$

Therefore $G$ satisfies (3.13) with $\bar{\mu}$ independent of $\phi$ if $-1<\phi \leq 0$. Hence if $F$ satisfies (3.12), we obtain a tangential gradient estimate on the solution of (1.1), (1.4) for such $\phi$, which is independent of the contact angle. A similar argument gives a uniform tangential gradient estimate for $0<\phi<1$. Thus we obtain a tangential gradient estimate for solutions of (1.1), (1.4) which does not depend on the contact angle if that angle is constant. An analogous result for the capillarity equation

$$
F[u]=\operatorname{div} \nu+n H(x, u)=0 \text { in } \Omega,
$$

where $H \in C^{1}(\bar{\Omega} \times \mathbf{R})$ and $H_{z}<0$, was given by Simon and Spruck [28].

Finally we show that the regularity of $\Omega$ can be relaxed to $\partial \Omega \in C^{2, \alpha}$ for some $\alpha \in(0,1)$. In this case, it follows from the methods of $[20]$ that there are positive constants $\varepsilon, \varepsilon_{1}, \varepsilon_{2}, C_{1}$ and a function $\rho \in C^{2, \alpha}(\Omega) \cap C^{3}(\Omega)$ such that

$$
\varepsilon d(x) \leq \rho(x) \leq d(x), \quad\left|D^{3} \rho(x)\right| \leq C_{1} \rho(x)^{\alpha-1} \quad \text { for all } x \in \Omega
$$

and $|D \rho| \geq \varepsilon_{1}$ on the set where $\rho<\varepsilon_{2}$. For $\phi$ a nonnegative $C^{\hat{3}}\left(\mathbf{R}^{n}\right)$ function with support in the unit ball and $\int_{\mathbf{R}^{n}} \phi=1$, we extend the normal field $\gamma$ to some boundary neighbourhood $\Sigma$ by

$$
\gamma(x)=\int_{\mathbf{R}^{n}} D d(x-\rho(x) z) \phi(z) d z .
$$

If $\Sigma$ is sufficiently small, then $|D d(x)-D d(y)| \leq C_{2}(\Omega)|x-y|$ in $\Sigma$, and hence

$$
|\gamma(x)-D d(x)| \leq \int_{\mathbf{R}^{n}}|D d(x-\rho(x) z)-D d(x)| \phi(z) d z \leq C_{2} \rho(x) \text { for } x \in \Sigma .
$$

Also we have

$$
\gamma^{j} D_{i} \gamma^{j}(x)=\int_{\mathbf{R}^{n}}\left[D_{i j} d(x-\rho(x) z) \gamma^{j}-D_{i} \rho(x) \gamma^{j} D_{j k} d(x-\rho(x) z) z^{k}\right] \phi(z) d z
$$

and a similar expression for $\gamma^{i} D_{i} \gamma^{j}(x)$, so $\left|\gamma^{j} D_{i} \gamma^{j}\right|,\left|\gamma^{i} D_{i} \gamma^{j}\right|=O(\rho)$. In the same way, we can show that $|D \gamma|,\left|\gamma^{k} D^{2} \gamma^{k}\right|=O(1)$ and $\left|D^{2} \gamma\right|=O\left(\rho^{\alpha-1}\right)$. Hence

$$
\begin{aligned}
& 4 F^{i j} D_{i k} u D_{j}\left(c^{k m}\right) D_{m} u \geq-C(\Omega)\left(\mu\left[\mathcal{E} C^{2}\right]^{1 / 2} \Lambda\left|D^{2} u\right| w_{T}^{1 / 2}+\Lambda\left|D^{2} u\right| \rho v\right), \\
& 2 F^{i j} D_{i j}\left(c^{k m}\right) D_{k} u D_{m} u \geq-C(\Omega)\left[\mu \mathcal{E}+\Lambda w_{T}^{1 / 2} v \rho^{\alpha-1}\right] .
\end{aligned}
$$


We now set

$$
w=w^{\prime}+\left(M_{1} / \alpha_{1}\right) u^{*}+\alpha_{2} M_{1} v\left(\rho+\rho^{1+\alpha} /(1+\alpha)\right)
$$

and look at a point $x_{0} \in \bar{\Sigma}$ where $w$ attains its maximum. As before, we can arrange for $x_{0} \in \Sigma$ if $M_{1}$ is sufficiently large. But then, at $x_{0}$,

$$
F^{i j} D_{i j} w \geq \alpha \alpha_{2} M_{1} v \rho^{\alpha-1}-c(\Omega) \Lambda w_{T}^{1 / 2} v \rho^{\alpha-1},
$$

which is positive if $M_{1}$ is large enough. This contradiction leads to the desired estimate in Theorem 3.4 and a similar analysis, using a suitable change of variables, applies in Theorem 3.3.

4. Hölder estimates for first derivatives. Hölder estimates for the first derivative of solutions of the boundary value problem (1.1), (1.2) follow from the boundary weak Harnack inequality, Lemma 2.1, in much the same way as the corresponding interior estimates are derived from the interior weak Harnack inequality in [30]. Since we may assume at this stage that the gradient has already been bounded, we formulate our result under hypotheses corresponding to $\mathrm{F}^{*}, \mathrm{~F}^{*}$ in [30].

THEOREM 4.1. Let $\partial \Omega \in C^{3}$ and $u \in C^{3}(\Omega) \cap C^{2}(\bar{\Omega})$ be a solution of $(1.1),(1.2)$ with $|u|+|D u| \leq K$ in $\Omega$. Suppose that $F$ and $G$ satisfy the struciure conditions:

$$
\begin{gathered}
\Lambda \leq \mu \lambda \\
F(x, z, p, 0) \leq \mu_{0} \lambda \\
\left|F_{p}\right|,(1+|r|)^{-\theta}\left|F_{z}\right|,(1+|r|)^{-\theta}\left|F_{x}\right| \leq \mu_{1} \lambda(1+|r|) \\
\chi \geq \beta_{0}, \quad|G|,\left|\frac{\partial G}{\partial X_{i}}\right| \leq \mu_{1} \beta_{0}, \quad i=1, \ldots, 2 n+1,
\end{gathered}
$$

for all $X \in \Gamma$ with $|z|+|p| \leq K$, where $\mu, \mu_{0}, \mu_{1}, \beta_{0}$ are positive constants and $\theta<1$. Then there are positive constants $\alpha$ and $C$ depending on $n, \mu, \mu_{0}, \mu_{1}, K, \theta$, and $\Omega$ such that

$$
[D u]_{\alpha ; \Omega} \leq C .
$$

Note that condition (4.2) is implied (at least for an equivalent problem) by either $\lambda$ being bounded away from zero or $F\left(X_{0}\right)=0$ for some $X_{0} \in \Gamma$. Condition (4.4) is simply a quantification of the fact that $G \in C^{1}\left(\Gamma^{\prime}\right)$ is oblique. Similarly conditions (4.1), (4.2), and (4.3) automatically hold for quasilinear elliptic $F$ with coefficients in $C^{1}\left(\bar{\Omega} \times \mathbf{R} \times \mathbf{R}^{n}\right)$.

PROOF. Because of the interior estimate [30, Theorem 5.1] (which clearly extends to positive $\theta<1$ ), it suffices to prove a Hölder estimate only at boundary points. By the usual flattening of $\partial \Omega$ near a point $y$, which we can take as the origin, we thus reduce our consideration to the half-ball $B^{+}=\left\{x \in \mathbf{R}^{n}|| x \mid<1, x_{n}>0\right\}$, with boundary condition (1.2) holding on the flat portion, $B^{0}=\left\{x \in \mathbf{R}^{n}|| x \mid<\right.$ $\left.1, x^{n}=0\right\}$. As in [30, Lemma 7.2], we introduce functions of the form

$$
w^{ \pm}=w_{l}^{ \pm}= \pm D_{l} u+\varepsilon v^{\prime} \quad(0<\varepsilon \leq 1),
$$

where $l=1, \ldots, n-1$ and $v^{\prime}=\left|D^{\prime} u\right|^{2}=\sum_{i=1}^{n-1}\left|D_{i} u\right|^{2}$. By differentiation, we obtain

$$
-2 \varepsilon C^{2}+F^{i j} D_{i j} w^{ \pm}+F^{i} D_{i} w^{ \pm}+2 \varepsilon v^{\prime} \delta F \pm\left(F_{z} D_{l} u+F_{x l}\right)=0
$$


Using the mean value theorem and conditions (4.1) and (4.2), we can then estimate from equation (1.1) itself, as in the discussion following Lemma 3.2,

$$
\left|D_{n n} u\right| \leq n \mu\left(\sum_{i+j<2 n}\left(D_{i j} u\right)^{2}\right)^{1 / 2}+\mu_{0}
$$

Now by the ellipticity of $F$,

$$
C^{2}=\sum_{k=1}^{n-1} F^{i j} D_{i k} u D_{j k} u \geq \lambda \sum_{i+j<2 n}\left(D_{i j} u\right)^{2},
$$

and hence using the condition (4.3), we obtain the differential inequalities

$$
-F^{i j} D_{i j} w^{ \pm} \leq \lambda C\left(\left|D w^{ \pm}\right|^{2}+1\right),
$$

where $C=C\left(n, \mu, \mu_{0}, \mu_{1}, \theta, M_{1}, \varepsilon\right)$. Furthermore, by differentiation of (1.2), we have on $B^{0}$,

$$
\left|G^{i} D_{i} w^{ \pm}\right| \leq \chi C
$$

for $C=C\left(n, \mu_{1}, M_{1}\right)$. We now choose $\rho$ in accordance with Lemma 2.1 and for $0<R<\frac{1}{8}$, set

$$
W_{R}^{ \pm}=W_{l, R}^{ \pm}=\sup _{G(\rho, R)} w_{l}^{ \pm}
$$

where as in $\S 2$,

$$
\begin{gathered}
G(\rho, R)=\left\{\left|x^{\prime}\right|<R, 0<x^{n}<\rho R\right\}, \\
(R)=G^{\prime}(\rho, R)=\left\{\left|x^{\prime}\right|<R, \rho R<x^{n}<3 \rho R / 2\right\} .
\end{gathered}
$$

Applying Lemma 2.1 to the functions $W_{4 R}^{ \pm}-w^{ \pm}$, we thus obtain

$$
\left(\frac{1}{G(2 R)} \int_{G(2 R)}\left(W_{4 R}^{ \pm}-w^{ \pm}\right)^{\kappa}\right)^{1 / \kappa} \leq C\left\{W_{4 R}^{ \pm}-W_{R}^{ \pm}+R\right\},
$$

where $\kappa=\kappa(n, \mu)>0$ and $C$ depends on the same quantities as in (4.7). Let us now set

$$
\omega(R)=\sum_{l=1}^{n-1} \operatorname{osc}_{G(\rho, R)} D_{l} u
$$

and sum the above inequalities over all of the functions $w_{l}^{ \pm}$. With $\varepsilon$ chosen sufficiently small, in particular $\varepsilon=1 / 10 n M_{1}$, we then deduce (similarly to the interior quasilinear case of Ladyzhenskaya and Ural'tseva described in [9, Chapter 13])

$$
\omega(4 R) \leq C\{\omega(4 R)-\omega(R)+R\},
$$

and hence by the standard argument [9, Lemma 8.23], we obtain for the tangential gradient $D^{\prime} u=\left(D_{1} u, \ldots, D_{n-1} u\right)$,

$$
\operatorname{osc}_{B_{R}^{+}} D^{\prime} u \leq C R^{\alpha}
$$

for all $R \leq 1$, where $C$ and $\alpha$ are positive constants depending on $n, \mu, \mu_{0}, \mu_{1}, M_{1}, \Omega$. A Hölder estimate for the full gradient at the boundary follows readily from (4.9). 
For, using the boundary condition (1.2) itself, we may infer (4.9) for $D_{n} u$ restricted to $B^{0}$, that is

$$
\operatorname{osc}_{B_{R}^{0}} D u \leq C R^{\alpha},
$$

where $B_{R}^{0}=\left\{\left|x^{\prime}\right|<R, x_{n}=0\right\}$ and $C$ and $\alpha$ depend on the same quantities as in (4.9). But then the corresponding estimate in $B_{R}^{+}$follows by applying the boundary weak Harnack inequality [9, Theorem 9.27] to the differential inequalities (4.6) for the functions

$$
w^{ \pm}= \pm D_{l} u+\varepsilon|D u|^{2}, \quad l=1, \ldots, n,
$$

used in the interior case [30, Theorem 5.1]. Combining the resultant estimate,

$$
\operatorname{osc}_{B_{R}^{+}} D u \leq C R^{\alpha}
$$

with the interior estimate [30, Theorem 5.1] then yields the global estimate (4.5).

We remark that the use of the boundary weak Harnack inequality $[9$, Theorem 9.27] can be replaced by elementary barrier considerations. Alternatively we can proceed directly from the tangential gradient estimate by more elaborate barrier arguments as in $[22, \S 5]$. By using the function $\rho$, described at the end of $\S 3$, to effect the boundary flattening and using Lemma 2.2 in place of Lemma 2.1, we see that Theorem 4.1 remains valid for $\partial \Omega \in C^{2, \eta}, \eta \in(0,1)$, with the constants $C$ and $\alpha$ depending also on $\eta$. Further, the difference quotient argument described after Lemma 3.1 shows that $u$ need only lie in $W^{2, q}(\Omega)$ for some $q>(2-\theta) n$. Also it is evident that the condition on $F_{x}$ and $F_{z}$ in (4.3) can be relaxed to

$$
F_{x}, F_{z}=o\left(|r|^{2}\right) \quad \text { as } r \rightarrow \infty
$$

in which case we must take $q>2 n$. In the case of two variables we may take $\theta=1$ in Theorem 4.1 and simply let $w=D_{1} u$ in its proof.

5. Second derivative bounds. Now we obtain bounds on the second derivative of solutions of the boundary value problem (1.1), (1.2). In the interior of $\Omega$, these follow from [30, Theorem 6.1] so again our main concern is with estimates near the boundary (although we provide an alternative derivation of the interior bound). The boundary estimate proceeds in two stages. First we obtain one-sided bounds on the pure tangential derivatives and then two-sided bounds on (essentially) the pure normal derivatives. The mixed derivatives are estimated through the boundary condition and then the equation itself can be used to bound all the derivatives. The tangential estimate is based on the method of $\S 3$ as well as Krylov's idea [11], already used in [27], of introducing new independent variables; moreover, the dependence of the estimates on various quantities must be displayed explicitly. The normal estimate is also more complicated here than in [27] although our underlying philosophy is to treat the boundary condition (1.2) as being essentially the condition $\gamma \cdot D u=0$. A new idea here is to use a boundary gradient estimate for the solution of a Dirichlet problem with $C^{1, \alpha}$ boundary data similiar to those given in $[10,15]$.

We begin by using Krylov's technique to give an alternative proof of a variant of the interior second derivative bound in [30, Theorem 6.1] (see also [30, Theorem 7.5]). 
LEMMA 5.1. Let $u \in C^{4}(\Omega)$ be a solution of $F[u]=0$ in $\Omega$ with $|u|+|D u| \leq K$ in $\Omega$. Suppose that $F$ satisfies the structure conditions:

$$
\begin{aligned}
& \Lambda \leq \mu \lambda ; \\
& |F(x, z, p, 0)| \leq \mu_{0} \lambda \\
& |r|\left|F_{p}\right|,\left|F_{z}\right|,\left|F_{x}\right| \leq \mu_{1} \lambda|r|^{2} \\
& \begin{aligned}
\mathcal{F}(X, Y) & =\frac{\partial^{2} F}{\partial X_{i} \partial X_{j}}(X) Y_{i} Y_{j} \\
& \leq \mu_{2} \lambda\left\{|r|^{3}|\bar{y}|^{2}+|r||q|^{2}+(|\bar{y}||r|+|q|)|s|\right\}
\end{aligned}
\end{aligned}
$$

for all $X=(x, z, p, r) \in \Gamma$ with $|z|+|p| \leq K,|r| \geq M$ and for all $Y=(y, w, q, s) \in$ $\mathbf{R}^{n} \times \mathbf{R} \times \mathbf{R}^{n} \times \mathbf{S}^{n}$ where $\bar{y}=(y, w)$ and $\mu, \mu_{0}, \mu_{1}, \mu_{2}$ and $M$ are nonnegative constants. Then there are constants $C$ and $\theta$ depending only on $K, M, n, \mu, \mu_{0}, \mu_{1}$ and $\mu_{2}$ such that if $B=B_{R}(y) \subset \Omega$ and $\alpha=\operatorname{osc}_{B} D^{\prime} u<\theta$, where $D^{\prime} u=$ $\left(D_{1} u, \ldots, D_{n-1} u\right)$, then

$$
\sup _{B} \eta_{R}\left|D^{2} u\right| \leq C \alpha / R+\mu_{0}+M
$$

where $\eta_{R}(x)=\left(1-|x-y|^{2} / R^{2}\right)^{2}$. Further, the estimate (5.5) remains valid if $B$ is replaced by $B \cap \Omega$ for any ball $B=B_{R}(y)$ with $y \in \Omega$ and $\left|D^{2} u\right| \leq M$ on $\partial \Omega \cap B$.

ProOF. For $\xi \in \mathbf{R}^{n}$, set $u_{\xi}=\xi \cdot D u, Y_{\xi}=\left(\xi, u_{\xi}, D u_{\xi}, D^{2} u_{\xi}\right), \tilde{w}=\tilde{w}(x, \xi)=$ $D_{i j} u(x) \xi^{i} \xi^{j}, \eta(x, \xi)=1-|x|^{2} / R^{2}-|\xi|^{2}, \tilde{M}_{2}=\sup _{B_{R} \times B_{1}} \eta \tilde{w}$, and $M_{2}=$ $\sup _{B_{R} \times B_{1}} \eta_{R} \tilde{w}$. Without loss of generality we can assume that $y=0, D u(y)=0$ and $\alpha \leq 1$. We then set

$$
w=\eta \tilde{w}+\tilde{M}_{2} v^{\prime} / 2 \alpha^{2}, \quad v^{\prime}=\sum_{k=1}^{n-1}\left|D_{k} u\right|^{2} .
$$

Our first step is to relate $\tilde{M}_{2}$ and $M_{2}$. Maximizing over $\xi$, we have

$$
D_{\xi}(\eta \tilde{w})=-2 \xi^{i} \tilde{w}+2 \eta D_{i j} u \xi^{j}=0
$$

and hence

$$
\xi^{i} \tilde{w}=\eta D_{\imath j} u \xi^{j}, \quad|\xi|^{2}=\eta, \quad \eta_{R}=4|\xi|^{4}
$$

and therefore $\tilde{M}_{2}=M_{2} / 4$. Furthermore it follows from (5.1), (5.2), that for each $x \in \Omega$,

$$
\left|D^{2} u(x)\right| \leq(n-1) \mu \sup _{|\xi|=1} \tilde{w}+\mu_{0}+M
$$

so that the estimate on $\eta_{R}\left|D^{2} u\right|$ follows from an estimate on $\tilde{M}_{2}$. We now observe (see $[30,(6.3)])$ that

$$
F^{i j} D_{i j} \tilde{w}=-\mathcal{F}\left(X, Y_{\xi}\right)-F^{i} D_{i} \tilde{w}-F_{z} \tilde{w}
$$

so with $B^{i}$ given by $B^{i}=F^{i}-(2 / \eta) F^{i j} D_{j} \eta$, we have

$$
\begin{aligned}
F^{i j} D_{i j} w+B^{i} D_{i} w= & \alpha^{-2} \tilde{M}_{2} C^{2}-\eta \mp+\tilde{w}\left(-F_{z} \eta+F^{i} D_{i} \eta-F^{i j} D_{i j} \eta\right) \\
& -\alpha^{-2} \tilde{M}_{2}\left(F_{z} v^{\prime}+F_{k} D_{k} u+\frac{2}{\eta} F^{i j} D_{i} \eta D_{j k} u D_{k} u\right)
\end{aligned}
$$


where summation over $k$ runs from 1 to $n-1$. In order to control the third derivatives of $u$ occurring in $₹$ on the right-hand side of (5.9), we set

$$
A^{i j}=-\lambda \mu_{2} \sqrt{1+K^{2}}|\xi|\left|D^{2} u\right| \operatorname{sign}\left(D_{i j} u_{\xi}\right)
$$

so that by (5.4),

$$
\mathcal{F} \leq-2 A^{i j} D_{i j} u_{\xi}+\lambda \mu_{2}\left(2+K^{2}\right)|\xi|^{2}\left|D^{2} u\right|^{3}
$$

for $\left|D^{2} u\right| \geq M$. Using the remaining structure conditions (5.1), (5.2), (5.3) in (5.9), together with (4.6) and the definition of $\alpha$, we then obtain for $\left|D^{2} u\right| \geq M$ and $\eta \tilde{w} \geq \tilde{M}_{2} / 2$, the differential inequality

$$
F^{i j} D_{i j} w+2 \eta A^{i j} D_{i j m} u \xi^{m} \geq \tilde{M}_{2} C^{2}\left\{\frac{1}{\alpha^{2}}-C_{1}\left(1+\frac{1}{\alpha R \tilde{M}_{2}}+\frac{1}{\left(R \tilde{M}_{2}\right) 2}\right)\right\}
$$

where $C_{1}$ is a constant depending on $n, K, M, \mu_{0}, \mu_{1}$ and $\mu_{2}$. Moreover if $\tilde{M}_{2} \geq$ $3 C_{1} \alpha / R, \alpha<1 / \sqrt{2 C_{1}}$, we have

$$
F^{i j} D_{i j} w+2 \eta A^{i j} D_{i j m} u \xi^{m} \geq \tilde{M}_{2} C^{2} / 2 \alpha^{2} .
$$

Since we are maximizing with respect to $x$ and $\xi$ we introduce some appropriate notation. Namely, we write $D_{i+n}$ for $\partial / \partial \xi^{i}, i=1, \ldots, n$, and we let $\sigma, \tau$ be indices running from 1 to $2 n$. We need to show that if $\tilde{M}_{2}>C \alpha / R$ and $\alpha$ is sufficiently small, then $G^{\sigma \tau} D_{\sigma \tau} w>0$ for some positive matrix $\left[G^{\sigma \tau}\right]$. By calculation

$$
\begin{aligned}
& D_{i+n, j} w=\frac{2}{n} D_{j} \eta \tilde{w} \xi^{i}-2 \xi^{i} D_{j} \tilde{w}+2 \eta D_{i j m} u^{\xi} \\
& D_{i+n, j+n} w=-2\left(\xi^{i} D_{j m} u \xi^{m}+D_{i m} u \xi^{m} \xi^{j}\right)-2 \delta^{i j} \tilde{w}+2 \eta D_{i j} u
\end{aligned}
$$

so if

$$
G^{\sigma \tau}= \begin{cases}F^{i j}, & \sigma=1, \tau=j \\ A^{i j}, & \sigma=i, \tau=j+n \text { or } \sigma=j+n, \tau=i \\ \tilde{G} \delta^{\sigma \tau}, & \sigma, \tau>n\end{cases}
$$

for some positive $\tilde{G}$, then

$$
\begin{aligned}
G^{\sigma \tau} D_{\sigma \tau} w= & F^{i j} D_{i j} w+\eta A^{i j} D_{i j m} u \xi^{m}-A^{i j} D_{i} \eta D_{j m} u \xi^{m}-\frac{1}{\eta} A^{i j} \xi^{i} D_{j} w \\
& +\frac{1}{\eta} A^{i j} \xi_{i}\left(D_{j} \eta\right) \tilde{w}+\frac{1}{\alpha^{2} \eta} A^{i j} \xi^{i} D_{j k} u D_{k} u+\tilde{G}(-8 \tilde{w}-2 n \tilde{w}+2 \eta \Delta u) .
\end{aligned}
$$

We now choose $\tilde{G}$ to make $\left[G^{\sigma \tau}\right]$ positive; in particular we take $\tilde{G}=\sum\left|A^{i j}\right|^{2} / \lambda$. Consequently at a maximum point $\left(x_{0}, \xi_{0}\right)$ of $w$, we have $D w=0$ and therefore

$$
G^{\sigma \tau} D_{\sigma \tau} w \geq \tilde{M}_{2} C^{2}\left\{\frac{1}{2 \alpha^{2}}-C_{2}\left(1+\frac{1}{\alpha \tilde{M}_{2} R}\right)\right\}
$$

provided our previous restrictions apply, where now $C_{2}$ depends only on $n, K$ and $\mu_{2}$. Finally we deduce $G^{\sigma \tau} D_{\sigma \tau} w>0$ at $\left(x_{0}, \xi_{0}\right)$ for $\tilde{M}_{2}>C \alpha / R, \alpha<\theta$ if $C$ and $\theta$ are chosen appropriately in terms of the indicated quantities in the statement of Lemma 5.1, which is thus proved.

We remark that conditions (5.1), (5.2) in Lemma 5.1 are precisely (4.1), (4.2) in Theorem 4.1 but that (5.3) is more general than (4.3). Condition (5.4) is the 
corrected version of condition $\mathrm{F} 4^{*}$ in $[30]$ (which is clearly incorrect as presently written). If we replace $D^{\prime} u$ by the full gradient $D u$, which is adequate for our purposes here, then the condition (5.2) becomes superfluous [30, Theorem 6.1]. However the argument given above is useful in the present context as our one sided tangential situation boundary estimates are proved by adjustments analogous to the first derivative situation in $\S 3$. Moreover with $D^{\prime} u$ replaced by $D u$, and $C^{2}$ replaced by $\varepsilon_{2}$, the above proof extends to embrace nonuniformly elliptic equations, subject to the more general structure conditions

$$
\begin{aligned}
& \Lambda|r|^{2} \leq \mu \mathcal{E}_{2} \\
& F(x, z, p, 0) \leq \mu_{0} \lambda(x, z, p, 0) \\
& |r|\left|F_{p}\right|, F_{z},\left|F_{x}\right| \leq \mu_{1} \mathcal{E}_{2} \\
& \mathcal{F}(X, Y) \leq \mu_{2}\left\{\Lambda\left(|r|^{3}|\bar{y}|^{2}+|r||q|^{2}\right)(\lambda \Lambda)^{1 / 2}(|\bar{y}||r|+|q|)|s|\right\} .
\end{aligned}
$$

Furthermore if $\mu_{1}$ (except for the $F_{p}$ bound) and $\mu_{2}$ are sufficiently small, the estimate (5.5) does not require a restriction on $\alpha=\operatorname{osc}_{B} D u$. It is also evident from the proof of Lemma 5.1 that conditions (5.3), (5.4) (or $\left.(5.3)^{\prime},(5.4)^{\prime}\right)$, need only hold for $r_{i j} y_{i} y_{j} \geq M|y|^{2}$ rather than $|r| \geq M$ as asserted and $\left|F_{z}\right|$ may be replaced by $F_{z}$. These observations are also applicable to the study of obstacle problems.

The pointwise relation (5.8) between the maximum and minimum eigenvalues of the Hessian $\left[D^{2} u\right]$ plays a crucial role in the proof of Lemma 5.1. For our tangential second derivative estimates, the corresponding relation, which is no longer pointwise, is not proved so readily because $\xi$ ranges over a smaller set of directions. To overcome this difficulty we prove (in Lemma 5.3) estimates on other derivatives. For now, however, we assume the appropriate analogue of (5.8). When we consider tangential derivatives, as in the next lemma, we shall identify $\mathbf{R}^{n-1}$ with the set $\left\{\xi \in \mathbf{R}^{n} \mid \xi^{n}=0\right\}$. We also recall the definitions of $B_{R}^{+}$and $B_{R}^{0}$ from $\S 3$.

LEMMA 5.2. Let $u \in C^{3}\left(B^{+} \cup B^{0}\right) \cap C^{4}\left(B^{+}\right)$satisfy $F[u]=0$ in $B^{+}, G[u]=0$ on $\partial B^{+}$with $|u|+|D u| \leq K$ in $B^{+}$and for $R \in(0,1)$ set

$$
M_{2}^{+}(R)=\sup _{B_{R}^{+} \times \mathbf{R}^{n-1}} \eta_{R} D_{i j} u \xi^{i} \xi^{j} /|\xi|^{2}, \quad M_{2}(R)=\sup _{B_{R}^{+}} \eta_{R}\left|D^{2} u\right| .
$$

Suppose there are constants $\mu_{3}$ and $M(R)$ such that

$$
M_{2}(R) \leq \mu_{3} M_{2}^{+}(R)+M(R)
$$

and that there is a continuous increasing function $\omega$ with $\omega(0)=0$ for which

$$
\left|D^{\prime} u(x)-D^{\prime} u(0)\right| \leq \omega(|x|), \quad\left|D^{2} u(x)\right| \leq \omega(|x|) / x^{n} \quad \text { for } x \in B^{+} .
$$

Suppose also that $F$ satisfies the structure conditions (5.1), (5.2), (5.3), (5.4) with $\Omega=B^{+}$while $G$ satisfies

$$
\left|\frac{\partial G}{\partial X_{i}}\right| \leq \mu_{1} \chi, \quad \frac{\partial^{2} G}{\partial X_{i} \partial X_{j}} Y_{i} Y_{j} \leq \mu_{2} \chi|Y|^{2}
$$

for all $X=(x, z, p)$, with $x \in B^{0},|z|+|p| \leq K$ and $Y \in \mathbf{R}^{2 n+1}$. Then there are constants $R_{0} \in(0,1)$ depending only on $K, \mu, \mu_{0}, \mu_{1}, \mu_{2}, \mu_{3}, n, \omega$ and $C$, depending also on $R$ and $M(R)$, such that for each $R \in\left(0, R_{0}\right]$, we have

$$
M_{2}^{+}(R) \leq C \text {. }
$$


ProOF. For $\xi \in \mathbf{R}^{n-1}, \alpha_{1}, \alpha_{2}, R$ positive constants, define $v^{\prime}, \eta, \tilde{w}$ and $\tilde{M}_{2}$ as in Lemma 5.1 (with the supremum in the definition of $\tilde{M}_{2}$ now over $B_{R}^{+} \times\{|\xi|=$ $\left.\left.1, \xi^{n}=0\right\}\right)$. Then set

$$
\bar{\eta}=\eta+\alpha_{2} \tilde{M}_{2} x^{n}|\xi|^{2}, \quad w=\bar{\eta} \tilde{w}+\alpha_{1} \tilde{M}_{2} v^{\prime}
$$

and choose $R_{0}$ so small that

$$
\alpha_{2} x^{n} \tilde{w}|\xi|^{-2} \leq \frac{1}{4}, \quad \alpha_{1}\left(v^{\prime}\right)^{1 / 2} \leq \frac{1}{4}, \quad v^{\prime} \leq 1
$$

for $x \in B_{R_{0}}^{+}, 0<|\xi| \leq 1$, and let $R \in\left(0, R_{0}\right]$. At a maximum of $w$, it is readily seen that $\eta \tilde{w} \geq \tilde{M}_{2} / 2$ and that

$$
\begin{gathered}
\bar{\eta} D_{i k} u \xi^{k}=\left(1+\alpha_{2} \tilde{M}_{2} x^{n}|\xi|^{-4}\right) \tilde{w} \xi^{i} \quad \text { for } i=1, \ldots, n-1, \\
\bar{\eta}=|\xi|^{2}+\alpha_{2} M_{2} x^{n}|\xi|^{-2}
\end{gathered}
$$

so $\eta / 2 \leq|\xi|^{2} \leq 3 \eta / 2$.

Now if $\tilde{M}_{2}>R^{-1}+M(R)$, then, as in Lemma 5.1 , there is a constant $\alpha_{0}$ depending on $K, \mu, \mu_{0}, \mu_{1}, \mu_{2}, \mu_{3}, n, \alpha_{2}$ such that for $\alpha_{1} \geq \alpha_{0}$ we have

$$
F^{i j} D_{i j} w+2 \eta A^{i j} D_{i j k} u \xi^{k} \geq\left(\alpha_{1} / 2\right) \tilde{M}_{2} C^{2}
$$

at a maximum of $w$. Since

$$
D_{n n k} u \xi^{k}=-\frac{1}{F^{n n}}\left\{\sum_{i+j<2 n} F^{i j} D_{i j k} u \xi^{k}+F^{i} D_{i k} u \xi^{k}+F_{z}(D u \cdot \xi)+F_{x} \cdot \xi\right\}
$$

we may, by suitable redefinition of $A^{i j}$, assume that $A^{n n}=0$. By increasing $\alpha_{1}$ as needed, we then infer that $w$ cannot attain its maximum where $x^{n}>0$ if $\tilde{M}_{2}>1 / R+M(R)$.

We now consider a boundary maximum for $w$. On $B^{0} \times \mathbf{R}^{n-1}$, we have

$$
G^{i} D_{i} w=\left(G^{i} D_{i} \eta\right) \tilde{w}+\eta G^{i} D_{i} \tilde{w}+\alpha_{1} \tilde{M}_{2} G^{i} D_{i} v^{\prime}+\alpha_{2} \tilde{M}_{2} \tilde{w}|\xi|^{-2} \chi
$$

Since $G^{i} D_{i} v^{\prime}=-G_{z} v^{\prime}-G_{x_{k}} D_{k} u$, and $\tilde{w}|\xi|^{-2} \geq \tilde{M}_{2} / 2>\frac{1}{2}$ it follows that

$$
G^{i} D_{i} w>\left(\alpha_{2}-C\left(K, \mu_{1}, n\right)\right) \tilde{M}_{2} \tilde{w}|\xi|^{-2} \chi+G^{i} D_{i} \tilde{w}
$$

provided $\tilde{M}_{2}>R^{-1}+M(R)$. We now differentiate the equation $G[u]=0$ twice in the tangential direction $\xi$ to obtain

$$
\begin{aligned}
-G^{i} D_{i} \tilde{w}= & G^{i, j} D_{i k} u \xi^{k} D_{j m} u \xi^{m}+2 G_{z}^{i} D_{i k} u \xi^{k}(D u \cdot \xi)+2 G_{x}^{i} \cdot \xi D_{i k} u \xi^{k} \\
& +G_{z z}(D u \cdot \xi)^{2}+2\left(G_{z x} \cdot \xi\right)(D u \cdot \xi)+G_{i j} \xi^{i} \xi^{i}+G_{z} \tilde{w}
\end{aligned}
$$

Now from (5.14) and the first structure condition in (5.11), we have

$$
\left|D_{n k} u \xi^{k}\right| \leq \mu_{1}\left(\sum_{i<n}\left|D_{i k} u \xi^{k}\right|+(K+1)|\xi|\right)
$$

while from (5.13) we have

$$
\alpha_{2} \tilde{M}_{2} x^{n}|\xi|^{-4} \leq 2 \alpha_{2} \eta \tilde{w} x^{n}|\xi|^{-4} \leq 1 .
$$


Combining these inequalities with (5.15) and the second structure condition in (5.11) (together with the estimate $\eta^{2} \leq 1 \leq \tilde{w}^{2}$ ), we therefore infer that

$$
\eta G^{i} D_{i} \tilde{w} \geq-C\left(K, \mu_{1}, \mu_{2}, n\right) \eta \tilde{w}^{2}|\xi|^{-2} \chi
$$

Consequently

$$
G^{i} D_{i} w>\left(\alpha_{2}-C\right) M_{2}^{+} \tilde{w}|\xi|^{-2} \chi \geq 0
$$

for $\alpha_{2}=C$ and thus $w$ cannot attain its maximum on $B^{0} \times \mathbf{R}^{n-1}$, and the lemma is proved.

Of course, by virtue of the condition (5.10), we infer immediately a bound on $\eta_{R}\left|D^{2} u\right|$ and, in particular, on $\left|D^{2} u(0)\right|$ under the hypotheses of this lemma. By means of a more careful analysis of its prcof we can improve the form of the estimate on $M_{2}^{+}$. In particular, if the condition (5.4) is strengthened to

$$
\mathcal{F}(X, Y) \leq \mu_{2} \lambda\left(|r||\bar{y}|^{2}+|\bar{y}||s|\right)
$$

(so that $F$ becomes jointly convex with respect to $p$ and $r$ ), then the hypothesis (5.10) can be dispensed with, and the estimate (5.13) is independent of $\mu_{3}$ and $M(R)$. We also note here that the condition (5.11) is merely a one-sided quantification of the statement $G \in C^{2}$.

To complete our second derivative estimation, we need to establish (5.10). As a preliminary result, we derive a bound on $G^{i} D_{i n} u$, which is the correct analogue of $D_{n n} u$ for the nonlinear boundary condition. Because of the nonlinearity of $G$ the method of [27] is inadequate here and in its place we employ a subtle argument based on the boundary gradient estimates on [10 and $\mathbf{1 5}]$.

LEMMA 5.3. Let $u \in C^{2}\left(B^{+} \cup B^{0}\right) \cap C^{3}\left(B^{+}\right)$satisfy $F[u]=0$ in $B^{+}, G[u]=0$ on $B^{0},|u|+|D u| \leq K$, and for $R<1$, set

$$
M^{\prime}(R)=\sup _{B^{0} \cap B_{R} \times \mathbf{R}^{n-1}} \eta_{R}\left|D_{i j} u \xi^{i} \xi^{j}\right| /|\xi|^{2} .
$$

Suppose $F$ satisfies the structure conditions (5.1),(5.2), (5.3) with $\Omega=B^{+}$while $G$ satisfies

$$
\chi \geq \beta_{0} ; \quad|G|,\left|\frac{\partial G}{\partial X_{i}}\right| \leq \mu_{1} \beta_{0} ; \quad \frac{\partial^{2} G}{\partial X_{i} \partial X_{j}} Y_{i} Y_{j} \geq-\mu_{2} \beta_{0}|Y|^{2}
$$

for all $X=(x, z, p)$ with $x \in B^{0},|z|+|p| \leq K$, and all $Y \in \mathbf{R}^{2 n+1}$. Then, if $\sigma$ and $\delta_{1}$ are positive constants satisfying $\sigma \leq 1$ and

$$
\left|D^{\prime} u(x)-D^{\prime} u(y)\right| \leq \delta_{1}|x-y|^{\sigma} \quad \text { for all } x, y \in B^{0},
$$

there exists a constant $R_{0} \in(0,1)$ depending only on $\sigma, \delta_{1}, n, \mu$, and $\mu_{1}$, such that for any $R \leq R_{0}$ and arbitrary $\varepsilon>0$, we have

$$
\frac{1}{\beta_{0}} \sup _{B_{0}} \eta_{R} G^{i} D_{i n} u \leq \varepsilon M^{\prime}(R)+C_{\varepsilon}
$$

where $C_{\varepsilon}$ depends on $\varepsilon, \sigma, \delta_{1}, R, K, \mu, \mu_{0}, \mu_{1}, \mu_{2}, M$ and $n$.

Proof. With $R_{0}$ to be determined and $R \leq R_{0}$ fixed, we choose $y \in B^{0}$ so that $\eta_{R} G^{i} D_{i n} u$ attains its maximum over $B^{0}$ at $y$. Setting $w=(g+\tilde{\mu} \bar{v}) \eta_{R}$, where $g(x)=G\left(x^{\prime}, u(x), D u(x)\right), \bar{v}(x)=\left|D^{\prime} u(x)-D^{\prime} u(y)\right|^{2}$ and $\tilde{\mu}$ is a constant to be 
determined, we shall apply a barrier argument to estimate the normal derivative of $w$ at $y$. First we observe that, for $i, j=1, \ldots, n-1$ and $x \in B_{R}^{0}$, we have

$$
\begin{aligned}
\left|D_{i}\left(\eta_{R}(x)\left[D_{j} u(x)-D_{j} u(y)\right]\right)\right| & =\left|D_{i} \eta_{R}(x)\left[D_{j} u(x)-D_{j} u(y)\right]+\eta_{R}(x) D_{i j} u(x)\right| \\
& \leq \frac{4 \delta_{1}}{R}|x-y|^{\sigma}+M^{\prime} \leq \frac{1}{R}+M^{\prime}
\end{aligned}
$$

if $\delta_{1} R_{0}^{\sigma} \leq \frac{1}{4}$. Since $g=0$ on $B^{0}$, it therefore follows that

$$
\begin{aligned}
w(x) & \leq \tilde{\mu}\left(\eta_{R}\left|D^{\prime} u-D^{\prime} u(y)\right|\right)^{\tau} \eta_{R}^{1-\tau} \bar{v}^{1-\tau / 2} \\
& \leq \tilde{\mu} n^{2 \tau}\left(1 / R+M^{\prime}\right)^{\tau} \delta_{1}^{2-\tau}|x-y|^{2 \sigma+\tau-\sigma \tau}
\end{aligned}
$$

for $x \in B_{R}^{0}$ and any $\tau \in(0,1)$. In particular for $\tau=(4-7 \sigma) /(4-4 \sigma), \sigma \leq 4 / 7$, we have $2 \sigma+\tau-\sigma \tau=1+\sigma / 4$ and hence

$$
w(x) \leq \tilde{\mu} C_{1}\left(\delta_{1}, n, R, \sigma\right)\left(1+M^{\prime}\right)^{\tau} \cdot|x-y|^{1+\sigma / 4}
$$

for $x \in B_{R}^{0}$. We now need a suitable differential inequality for $w$ on $B_{R}^{+}$which we get by estimating $F^{i j} D_{i j} w$. First from the structure conditions (5.17), it follows that

$$
\begin{aligned}
F^{i j} D_{i j} g & \geq-C\left(K, \mu_{1}, \mu_{2}, n\right) \beta_{0} \Lambda\left(1+\left|D^{2} u\right|^{2}\right), \\
|D g| & \leq C\left(K, \mu_{1}, n\right) \beta_{0}\left(1+\left|D^{2} u\right|\right),
\end{aligned}
$$

so utilizing (5.1), (5.2) (or rather the consequent bound (4.6)), we obtain

$$
F^{i j} D_{i j}\left(\eta_{R} g\right) \geq-\bar{\mu} \beta_{0}\left(\eta_{R} C^{2}+R^{-2}\right)
$$

where $\bar{\mu}$ depends on $K, \mu, \mu_{0}, \mu_{1}, \mu_{2}, n$ and $M$. Furthermore by (5.1), (5.2), (5.3)

$$
\begin{aligned}
F^{i j} D_{i j} \bar{v} & =2 C^{2}-F^{i} D_{i k} u\left(D_{k} u-D_{k} u(y)\right)-F_{z} \bar{v}-F_{k}\left(D_{k} u-D_{k} u(y)\right) \\
& \geq 2 C^{2}-\left(2 \delta_{1}|x-y|^{\sigma}+\left(\delta_{1}|x-y|^{\sigma}\right)^{2}\right) \mu_{1}\left(\left|D^{2} u\right|^{2}+1\right) \Lambda \\
& \geq \frac{3}{2} C^{2}-\Lambda
\end{aligned}
$$

if $R$ is chosen sufficiently small, in terms of $n, \mu, \mu_{0}, \mu_{1}, \delta_{1}$ and $\sigma$. Since $\left|F^{i j} D_{j} \bar{v}\right| \leq$ $2\left(\Lambda C^{2}\right)^{1 / 2}$, we infer that

$$
F^{i j} D_{i j}\left(\eta_{R} \bar{v}\right) \geq \eta_{R} C^{2}-C R^{-2} \Lambda
$$

and therefore for $\tilde{\mu}=\bar{\mu} \beta_{0}$, we have

$$
F^{i j} D_{i j} w \geq-C_{2} \Lambda
$$

where $C_{2}=C_{2}\left(K, \mu, \mu_{0}, \mu_{1}, \mu_{2}, n, M, R\right)$. Next we set $\phi(x)=|x-y|^{1+\sigma / 4}$, and note that $\phi \in C^{0}\left(\bar{B}_{R}^{+}\right) \cap C^{2}\left(B_{R}^{+}\right)$and $|D \phi|+\left(x^{n}\right)^{1-\sigma / 4}\left|D^{2} \phi\right| \leq \Phi$ for some constant $\Phi=\Phi(n, \sigma)$. Hence for

$$
\bar{w}=\phi+4 \mu(1+\Phi)\left(x^{n}-\left(x^{n}\right)^{1+\sigma / 4}\right) / \sigma
$$

we have $F^{i j} D_{i j} \bar{w} \leq-\Lambda$ in $B_{R}^{+}$. Finally we set

$$
\mu^{*}=\left(\bar{\mu} C_{1}\left(1+M^{\prime}\right)^{\tau}+C_{2}\right) \beta_{0}
$$

and note that $w=0$ on $\partial B_{R} \cap B^{+}, \bar{w} \geq 0$ in $B^{+}$. Hence

$$
\begin{aligned}
F^{i j} D_{i j}\left(w-\mu^{*} \bar{w}\right) \geq 0 & \text { in } B_{R}^{+}, \\
w-\mu^{*} \bar{w} \leq 0 & \text { on } \partial\left(B_{R} \cap B^{+}\right)
\end{aligned}
$$


so the maximum principle implies that $w \leq \mu^{*} \bar{w}$ in $B_{R}^{+}$. Therefore, in particular, $D_{n} w(y) \leq \mu^{*} D_{n} \bar{w}(y)$. Upon computing $D_{n} \bar{w}(y)$, we see that

$$
D_{n} w(y) \leq C\left(1+M^{\prime}\right)^{\tau} \beta_{0} .
$$

Now using the fact that $\bar{v}, D_{n} \bar{v}$, and $g$ vanish at $y$, we obtain

$$
\begin{aligned}
D_{n} w(y) & =\eta_{R}\left(G^{i} D_{i n} u+G_{z} D_{n} u\right)(y) \\
& \geq \eta_{R} G^{i} D_{i n} u(y)-\mu_{1} K \beta_{0} .
\end{aligned}
$$

The desired estimate follows from (5.20) and (5.21) by recalling the choice of $y$ and noting that for any $\theta>0$, we have $(1+M)^{\tau} \leq C(\tau, \theta)+\theta M^{\prime}$.

Note that the above proof uses the Hölder continuity of $D u$ in a crucial way, whereas only continuity is required for Lemmas 5.1 and 5.2. The hypotheses of Lemma 5.3 may be weakened slightly in that $\lambda$ can be replaced by $F^{n n}$ in $(5.1)$ and $\lambda|r|^{2}$ by $\mathcal{E}_{2}$ in (5.3). Note also the second derivative conditions in (5.11) and (5.17) involve opposite inequalities. Intersecting (5.11) and (5.17) yields the structure condition

$$
|G|,\left|\frac{\partial G}{\partial X_{i}}\right| \leq \mu_{1} \chi, \quad\left|\frac{\partial^{2} G}{\partial X_{i} X_{j}}\right| \leq \mu_{2} \chi, \quad i, j=1, \ldots, 2 n+1,
$$

for all $|z|+|p| \leq K$, which simply quantifies the hypothesis $G \in C^{2}\left(\Gamma^{\prime}\right)$. To see this, we observe that (5.22) implies $\left|\partial \log \chi / \partial X_{i}\right| \leq \mu_{2}$ for $|z|+|p| \leq K$, and hence

$$
\sup \chi \leq C\left(n, K, \operatorname{diam} \Omega, \mu_{2}\right) \inf \chi,
$$

so that (5.17) holds with $\beta_{0}=\inf \chi$ and appropriate $\mu_{1}, \mu_{2}$.

It is now a simple matter to infer our second derivative estimates.

THEOREM 5.4. Let $\partial \Omega \in C^{4}$ and let $u \in C^{3}(\bar{\Omega}) \cap C^{4}(\Omega)$ be a solution of the boundary value problem (1.1), (1.2) with $|u|+|D u| \leq K$ in $\Omega$. Suppose that $F$ and $G$ satisfy the structure conditions (5.1), (5.2), (5.3), (5.4), and (5.22). Then for any $\sigma \in(0,1)$,

$$
\sup _{\Omega}\left|D^{2} u\right| \leq C\left(K, \mu, \mu_{0}, \mu_{1}, \mu_{2}, n, \sigma, \Omega, M,[D u]_{\sigma ; \Omega}\right) .
$$

Furthermore if (5.3) is replaced by (4.3), we have

$$
\sup _{\Omega}\left|D^{2} u\right| \leq C\left(K, \mu, \mu_{0}, \mu_{1}, \mu_{2}, n, \Omega, \theta, M\right) \text {. }
$$

PROOF. From the last statement in Lemma 5.1, a standard flattening of the boundary argument, and the remarks following Lemma 5.3, it follows that we need only verify (5.10) under the other hypotheses of Lemmas 5.2 and 5.3. Moreover if we set

$$
\begin{aligned}
& M_{ \pm}=\sup _{B^{0} \times \mathbf{R}^{n}}\left( \pm \eta_{R} D_{i j} u \xi^{i} \xi^{j}\right) /|\xi|^{2} \\
& M_{ \pm}^{\prime}=\sup _{B^{0} \times \mathbf{R}^{n-1}}\left( \pm \eta_{R} D_{i j} u \xi^{i} \xi^{i}\right) /|\xi|^{2}
\end{aligned}
$$

then (5.8) will imply (5.10) provided we show that

$$
M_{+} \leq C_{1}+(1+\varepsilon) M_{+}^{\prime}+\varepsilon M_{-}^{\prime}
$$

for all $\varepsilon>0$, where $C_{1}$ is a constant depending on $\varepsilon$ and the quantities in (5.23). 
To prove (5.25) we choose $y \in B^{0}$ and $\xi \in \mathbf{R}^{n}$ with $|\xi|=1$ so that

$$
M_{+}=\eta_{R}(y) D_{i j} u(y) \xi^{i} \xi^{j} \text {. }
$$

Now if we define vectors $\zeta$ and $\bar{\zeta}$ by $\zeta^{n}=\bar{\zeta}^{n}=0$,

$$
\varsigma^{i}=\xi^{i}-\xi^{n} G^{i} / \chi, \quad \bar{\zeta}^{i}=\left(\xi^{n} / \chi\right)\left(\zeta^{i}+\xi^{i}\right), \quad i=1, \ldots, n-1,
$$

then $|\zeta|,|\chi \bar{\zeta}| \leq C\left(\mu_{1}\right)$ and $D_{i j} u \xi^{i} \xi^{j}=D_{i j} u \varsigma^{i} \zeta^{j}+D_{i j} u \bar{\zeta}^{i} G^{j}+\left(\left(\xi^{n}\right)^{2} G^{i} / \chi\right) D_{i n} u$ so that

$$
M_{+} \leq M_{+}^{\prime}+D_{i j} u \bar{\zeta}^{i} G^{j}+C_{2}+\varepsilon M_{+}^{\prime}+\varepsilon M_{-}^{\prime},
$$

where $C_{2}$ is the constant of Lemma 5.3. Since $\bar{\zeta}^{n}=0$, we have

$$
D_{i j} u \bar{\zeta}^{i} G^{j}=-G_{z} D u \cdot \bar{\zeta}-G_{x} \cdot \bar{\zeta} \leq C\left(\mu_{1}\right)|\bar{\zeta}| \chi \leq C\left(\mu_{1}\right) .
$$

Inserting this estimate in (5.26) gives (5.25). This proves the estimate (5.23). The last assertion in Theorem 5.4 then follows by combination with Theorem 4.1.

We close this section by pointing out that the regularity hypotheses in Theorem 5.4 can be relaxed. First, similarly to the situation in $§ 3$, we need only assume $\partial \Omega \in C^{3, \alpha}$ for some $\alpha>0$. Moreover, all the results in this section remain valid for $u \in C^{2}(\bar{\Omega})$.

To verify this last assertion, we first use standard difference quotient arguments $[9, \S \S 6.4$ and $17.5 ; 1, \S 11]$ along with the $L^{p}$ estimate $[1$, Theorem 15.1] to infer that $u \in W^{3, q}(\Omega) \cap W_{\text {loc }}^{4, q}(\Omega)$ for all $q>1$. This regularity clearly suffices for Lemmas 5.1 and 5.3. In Lemma 5.2 we replace $\tilde{w}$ by

$$
\tilde{w}_{h}(x, \xi)=[u(x+h \xi)+u(x-h \xi)-2 u(x)] / h^{2} .
$$

The argument of Lemma 5.2 is then modified according to the discussion after Lemma 3.1, taking into account that $\tilde{w} \rightarrow \tilde{w}$ in $W^{1, q}$.

Note that for $n=2$, Lemma 5.3 and the gradient estimates of Von Wahl [33], directly imply (5.23), (5.24) with $\theta=1$ for $\partial \Omega \in C^{3}$ and without the second derivative condition (5.4).

6. Hölder estimates for second derivatives. We arrive now at the final stage of our series of estimates. Assuming that we have bounded $u$ along with its first and second derivatives, we estimate the Hölder norm of the second derivatives. In outline, the derivation of this estimate is similar to that of the second derivative bounds in §5. A partial estimation of pure tangential derivatives is accomplished by a modification of known methods for estimating all pure second derivatives for the Dirichlet problem [9]. On combination with a full estimate on the quantities $G^{i} D_{i j} u,(j=1, \ldots, n)$, we deduce the complete bound for $D^{2} u$. A new complication is that our one-sided estimate involves the behavior of the second derivatives in a neighborhood of the boundary, while initially the two-sided estimate is valid only on the boundary. This difficulty was overcome in [27] by conversion to a divergence structure situation. Here we proceed under more general hypotheses by using the Schauder interior estimates, although the elimination of the hypothesis (6.3) below would be of some interest. As the nontangential derivative bounds can, unlike the case in Lemma 5.3, be dealt with independently of the one-sided tangential derivative bounds, we consider them first. 
LEMMA 6.1. Let $u \in C^{1,1}\left(B^{+} \cup B^{0}\right) \cap C^{4}\left(B^{+}\right)$be a solution of $F[u]=0$ in $B^{+}$, $G[u]=0$ on $B^{0}$, with $|u|+|D u|+\left|D^{2} u\right| \leq K$ in $B^{+}$and suppose there are positive constants $\alpha<1$ and $\beta_{0}$ such that, for $\Omega=B^{+}, F$ and $G$ satisfy the structure conditions

$$
\begin{aligned}
& \Lambda \leq \mu \lambda ; \quad\left|F_{p}\right|,\left|F_{z}\right|,\left|F_{x}\right| \leq \mu_{1} \lambda \\
& \frac{\partial^{2} F}{\partial X_{i} X_{j}} Y^{i} Y^{j} \leq \mu_{2} \lambda\left\{|\bar{y}|^{2}+|q|^{2}+(|\bar{y}|+|q|)|s|\right\} \\
& \left|\frac{1}{\lambda} \frac{\partial F}{\partial X}\right|_{\alpha ; \Gamma_{K}} \leq \mu_{3} ; \\
& G^{n} \geq \beta_{0} ; \quad\left|\frac{\partial G}{\partial X}\right| \leq \mu_{2} \beta_{0} ; \quad\left|\frac{\partial^{2} G}{\partial X_{i} \partial X_{j}}\right| \leq \mu_{2} \beta_{0} \\
& G^{i}(0)=\delta^{i n}
\end{aligned}
$$

for all $X \in \Gamma_{K}=\{(x, z, p, r)|x \in \Omega| z,|+| p|+| r \mid \leq K\}$ and for all $Y=(\bar{y}, q, s) \in$ $\mathbf{R}^{n+1} \times \mathbf{R}^{n} \times \mathbf{S}^{n}$. Then there are positive constants $\eta$ depending only on $n$ and $\mu$ and $C$ depending also on $K, n, \alpha, \mu, \mu_{1}, \mu_{2}, \mu_{3}$ such that for any $R \leq 1$

$$
\operatorname{osc}_{B_{R}^{+}} D_{i n} u \leq C R^{\eta}, \quad i=1, \ldots, n .
$$

PrOOF. Let us define, similarly to the proof of Lemma 5.3,

$$
g(x)=g\left(x^{\prime}, x^{n}\right)=\frac{1}{\beta_{0}} G\left(x^{\prime}, u(x), D u(x)\right)
$$

and set $a^{i j}=F^{i j} / \lambda, L=a^{i j} D_{i j}$. Then

$$
|L g| \leq C\left(K, n, \mu_{1}, \mu_{2}\right) \quad \text { in } B^{+}, \quad g=0, \quad \text { on } B^{0} .
$$

It then follows from Krylov's boundary Hölder estimate [12, Theorem 4.1], in particular inequality (2.14) in $\S 2$, that there is a constant $\eta=\eta(n, \mu) \in(0,1)$ for which the function $v$, given by $v(x)=g(x) / x^{n}$, satisfies

$$
\operatorname{osc}_{B_{R}^{+}} v \leq C R^{\eta} \text { for all } R \leq 1 \text {, }
$$

where now $C$ depends on $K, n, \mu_{1}, \mu_{2}$ and $\mu$. Next we observe that the interior second derivative Hölder estimate $[30,(6.16)]$ implies that for a further constant $\varepsilon=\varepsilon(n, \mu) \in(0,1)$, we have $\left|D^{2} u\right|_{\varepsilon ; B^{+}}^{*} \leq C$ whence by condition (6.3)

$$
\left|a^{i j}\right|_{\alpha \varepsilon ; B^{+}}^{*}+|L g|_{\alpha \varepsilon ; B^{+}}^{*} \leq C
$$

where now and henceforth $C$ depends on $K, n, \mu, \mu_{1}, \mu_{2}, \mu_{3}$ and $\alpha$, and the interior norms are as defined, for example, in $[9$, Chapter 4] or $[30, \S 2]$. Now we can apply the Schauder interior estimate [9, Theorem 6.2] to conclude, in particular, for any ball $B_{1}=B_{R}(y)$ with $B_{2}=B_{2 R}(y) \subset B^{+}$and any $\eta \in(0,1]$,

$$
R^{-\eta}[D g]_{\eta ; B_{1}}+[D g]_{0 ; B_{1}} \leq C\left(\frac{1}{R}|g|_{0 ; B_{2}}+R\right) .
$$

The combination of (6.7) and (6.8) readily yields a local boundary estimate

$$
[D g]_{\eta ; B_{1 / 2}^{+}} \leq C
$$


where $\eta=\eta(n, \mu) \in(0,1)[24]$ (see [9, Theorem 8.29]); however for our immediate purposes we need only use the gradient bound on (6.8) to deduce for any point $y \in B_{1 / 4}^{+} R=\frac{1}{2} y^{n}$,

$$
|D g(y)| \leq C\left(\frac{1}{R} \sup _{B_{R}(y)}|g|+R\right) \leq C|y|^{n}
$$

by (6.7), provided we normalize $v(0)=0$. Writing

$$
\beta_{0} D^{i} g=G^{n} D_{i n} u+\left(G^{j}-\delta^{j n} G^{n}\right) D_{i j} u+G_{z} D_{i} u+G_{x_{i}},
$$

the desired result then follows from the conditions (6.3), (6.4).

Note that since $F \in C^{2}, \lambda$ is automatically in $C^{0,1}$ and also by virtue of the discussion preceding the statement of Theorem 5.4, the conditions (6.4) may be replaced by the hypotheses

$$
\left|\frac{\partial G}{\partial p}\right| \leq \mu \chi, \quad\left|\frac{\partial G}{\partial z}\right|,\left|\frac{\partial G}{\partial x}\right| \leq \mu_{1} \chi ; \quad\left|\frac{\partial^{2} G}{\partial X_{i} X_{j}}\right| \leq \mu_{2} \chi
$$

while the condition (6.5) may be achieved through a linear coordinate change:

$$
x^{i} \rightarrow x^{i}-x^{n} G^{i}(0) / G^{n}(0), \quad i<n, x^{n} \rightarrow x^{n} .
$$

For equations in two variables, Lemma 6.1 (without the restrictions (6.2), (6.3)) follows from Morrey's Hölder estimate [9, Theorem 13.4] and, when adjoined to the corresponding interior estimate [9, Theorem 17.11], it implies a global second derivative Hölder estimate which we return to later in this section. In the general $n$-dimension case there are $n(n-1) / 2>1$ second derivatives not directly controlled via Lemma 6.1. To get appropriate control over these other derivatives we modify the proof of [30, Theorem 6.1] using our boundary weak Harnack inequality, Lemma 2.1 , and in this way obtain the global Hölder estimates.

THEOREM 6.2. Let $u \in C^{3}(\bar{\Omega}) \cap C^{4}(\Omega)$ be a solution of the boundary value problem (1.1), (1.2) with $|u|+|D u|+\left|D^{2} u\right| \leq K$ in $\Omega$, and suppose that $F$ and $G$ satisfy the structure conditions $(6.1),(6.2),(6.3),(6.4)^{\prime}$. Then there are positive constants $\eta=\eta(n, \mu)<1, C_{1}=C_{1}\left(K, n, \mu, \mu_{1}, \mu_{2}, \mu_{3}, \alpha, \Omega\right)$ and $C_{2}=$ $C_{2}\left(K, n, \mu, \mu_{1}, \mu_{2}, \operatorname{diam} \Omega\right)$ such that:

$$
\begin{aligned}
& {\left[D^{2} u\right]_{\eta ; \Omega} \leq C_{1}} \\
& {\left[D^{2} u\right]_{\eta ; \Omega^{\prime}} \leq C_{2}\left[\operatorname{dist}\left(\Omega^{\prime}, \partial \Omega\right)\right]^{-\eta}}
\end{aligned}
$$

for any $\Omega^{\prime} \subset \subset \Omega$.

PROOF. The Evans-Krylov interior estimate (6.11) is contained in [30, Theorem 6.1] so that we need only estimate the Hölder norm near $\partial \Omega$ which we may assume has been locally flattened with (6.1), (6.2), (6.3), (6.4) holding. Now for $(x, \xi) \in$ $B^{+} \times \mathbf{R}^{n-1}$, with $|\xi|=1$, we set $h(x, \xi)=D_{i j} u \xi^{i} \xi^{j}$ and note that by appropriate adjustment of $u$, we may assume without loss of generality that $0 \leq h \leq 1$. As in Lemma 6.1, we see that for any such $\xi$, the function $h$ satisfies

$$
-F_{i j} D_{i j} h \leq \lambda C\left(\sum_{\substack{i+j<2 n \\ k<n}}\left|D_{i j k} u\right|+1\right) \text {. }
$$


Now let $\xi_{1}, \ldots, \xi_{M}$ be unit vectors including $e_{i},\left(e_{i} \pm e_{j}\right) \sqrt{2}, i, j=1, \ldots, n-1$, where $e_{1}, \ldots, e_{n-1}$ denotes the canonical basis in $\mathbf{R}^{n-1}$. Set

$$
h_{k}(x)=h\left(x, \xi_{k}\right), \quad k=1, \ldots, M, \quad v^{\prime}=\sum_{k=1}^{M}\left(h_{k}\right)^{2}, \quad a^{i j}=F^{i j} / \lambda
$$

and for $\varepsilon \in(0,1)$ to be chosen later, set

$$
w_{k}=h_{k}+\varepsilon v^{\prime}, \quad k=1, \ldots, M .
$$

If we write $\Xi=\left\{\xi_{1}, \ldots, \xi_{M}\right\}$, then we obtain (similarly to the interior case [30, $(6.7)])$

$$
-a^{i j} D_{i j} w_{k} \leq \bar{\mu} \quad \text { in } B^{+}, \quad-G_{p} \cdot D w_{k} \leq \bar{\mu}_{1} \quad \text { on } B^{0}
$$

for $\bar{\mu}=C\left(K, n, \mu, \mu_{1}, \mu_{2}, M\right) / \varepsilon^{2}$ and $\bar{\mu}_{1}=C\left(n, K, \mu, \mu_{1}, \mu_{2}, M\right)(1+\varepsilon)$. Thus we are in a position to apply the weak Harnack inequality, Lemma 2.1. To do this we set, for $R \leq \frac{1}{2}, \rho=1 /(4 n \mu)$,

$$
\begin{aligned}
& \Sigma_{R}=G(\rho, R)=\left\{\left|x^{\prime}\right|<R, 0<x^{n}<\rho R\right\}, \\
& G^{\prime}=G^{\prime}(\rho, R)=\left\{\left|x^{\prime}\right|<R, \rho R<x^{n}<3 \rho R / 2\right\}
\end{aligned}
$$

and, for $s=1,2, k=1, \ldots, M$,

$$
W_{k}^{(s)}=\sup _{\Sigma_{s R}} w_{k}, \quad M_{k}^{(s)}=\sup _{\Sigma_{s R}} h_{k}, \quad m_{k}^{(s)}=\inf _{\Sigma_{s R}} h_{R}, \quad \omega^{\prime}(s R)=\sum_{k=1}^{M} \operatorname{osc}_{\Sigma_{s R}} h_{k} .
$$

Applying Lemma 2.1 to the functions $W_{k}^{(2)}-w_{k}$, we infer that there are constants $C$ and $\kappa$ depending only on $n, \mu$ such that

$$
\left(R^{-n} \int_{G^{\prime}}\left(W_{k}^{(2)}-w_{k}\right)^{\kappa}\right)^{1 / \kappa} \leq C\left(W_{k}^{(2)}-W_{k}^{(1)}+\bar{\mu} R^{2}+\bar{\mu}_{1} R\right) .
$$

Noting that

$$
\begin{aligned}
& W_{k}^{(2)}-w_{k} \geq M_{k}^{(2)}-M_{k}^{(1)}-2 \varepsilon K \omega^{\prime}(2 R) \\
& W_{k}^{(2)}-W_{k}^{(1)} \leq M_{k}^{(2)}-M_{k}^{(1)}+2 \varepsilon K \omega^{\prime}(2 R)
\end{aligned}
$$

we infer that

$$
\left(R^{-n} \int_{G^{\prime}}\left(M_{k}^{(2)}-h_{k}\right)^{\kappa}\right)^{1 / \kappa} \leq C_{1}\left(M_{k}^{(2)}-M_{k}^{(1)}+K \varepsilon \omega^{\prime}(2 R)+\bar{\mu} R^{2}+\bar{\mu}_{1} R\right)
$$

for some $C_{1}=C_{1}(n, \mu)$. Next we conclude from Lemma 6.1 that

$$
\operatorname{osc}_{B_{R}^{+}} D_{i n} u \leq \bar{\mu}_{2} R^{\theta}
$$

for some $\theta=\theta(n, \mu)$ and $\bar{\mu}_{2}=\bar{\mu}_{2}\left(\alpha, K, n, \mu, \mu_{1}, \mu_{2}, \mu_{3}\right)$. We now connect (6.12) and (6.13). By the Motzkin-Wasow lemma [9, Lemma 17.13], there are positive constants $\lambda^{*}, \Lambda^{*}, N$ and unit vectors $\zeta_{1}, \ldots, \zeta_{N}$ depending only on $n$ and $\mu$, and functions $\alpha_{k}, k=1, \ldots, N$, with $\lambda^{*} \leq \alpha_{k} \leq \Lambda^{*}$ such that

$$
a^{i j}=\sum_{k=1} \alpha_{k} \zeta_{k}^{i} \zeta_{k}^{j}
$$


moreover, we can take these vectors to include $e_{i}$ and $\left(e_{i} \pm e_{j}\right) / \sqrt{2}$ for $i, j=1, \ldots, n$, $i<j$, where $e_{i}$ is the $i$ th standard basis vector and $\zeta_{N}=e_{n}$. We now choose $M=N-1$ and, for $k=1, \ldots, N-1$, we set

$$
\begin{aligned}
& \xi_{k}=\zeta_{k}^{\prime} /\left|\zeta_{k}^{\prime}\right| \\
& g_{k}=g_{k}(x)=h\left(x, \xi_{k}\right)=\left|\zeta_{k}^{\prime}\right|^{2} h_{k}+2 \zeta_{k}^{n}\left|\zeta_{k}^{\prime}\right| \zeta_{k}^{j} D_{j n} u+\left(\zeta_{k}^{n}\right)^{2} D_{n n} u, \\
& \bar{g}_{k}=g_{k}-\left|\zeta_{k}^{\prime}\right|^{2} h_{k}, \\
& g_{N}=\bar{g}_{N}=h\left(x, \zeta_{N}\right)=D_{n n} u .
\end{aligned}
$$

Since $0<\left|\zeta_{k}^{\prime}\right| \leq 1$ for $k<N$, it follows from (6.14) that

$$
\left|\bar{g}_{k}(x)-\bar{g}_{k}(y)\right| \leq 4 \bar{\mu}_{2} R^{\theta} \quad \text { for } x, y \in G^{\prime}, k=1, \ldots, N \text {. }
$$

Now setting, for $s=1,2$,

$$
G_{k}^{(s)}=\sup _{\Sigma_{s R}} g_{k}, \quad g_{k}^{(s)}=\inf _{\Sigma_{s R}} g_{k}, \quad \omega(s R)=\sum_{k=1}^{N} \operatorname{osc}_{\Sigma_{s R}} g_{k},
$$

we conclude from (6.12), (6.14), and (6.15) that

$$
\begin{aligned}
& \left(R^{-n} \int_{G^{\prime}}\left(G_{k}^{(2)}-g_{k}\right)^{\kappa}\right)^{1 / \kappa} \\
& \quad \leq C_{1} K \varepsilon \omega(2 R)+C_{2}(n, \mu)\left(G_{k}^{(2)}-G_{k}^{(1)}+\bar{\mu} R^{2}+\bar{\mu}_{1} R+\bar{\mu}_{2} R^{\theta}\right) .
\end{aligned}
$$

The desired Hölder estimate (6.11) now follows from this inequality in the same way that $[30,(6.15)]$ follows from $[30,(6.9)]$.

We remark that it follows from linear regularity considerations, or in particular [23, Theorem 6], that the estimate (6.11) is valid for all $\eta<1$ with constant $C_{1}$ depending in addition on $\eta$. We also note that the regularity hypotheses in Theorem 6.2 can be relaxed. First of all, by virtue of Lemma 2.2, we need only assume that $\partial \Omega \in C^{3, \delta}$ for some $\delta>0$ and, as in $\S 5, u \in C^{2}(\bar{\Omega})$. More significantly, by invoking the regularity technique of $[\mathbf{3 1}]$ with variable directions, it suffices to only assume $u \in C^{1,1}(\bar{\Omega})$.

The combination of Theorems 2.4, 3.3, 4.1, 5.4 with Theorem 6.2 now yields the global estimate, Theorem 1.1, asserted in the introduction. Furthermore by virtue of our previous remarks concerning the two variable case, we may eliminate all dependence on the second derivatives of $F$ in this case, so that Theorem 1.1 holds for $F \in C^{1}(\Gamma)$ (even $C^{0,1}(\Gamma)$ ) satisfying F1, F2, F3 with $\partial \Omega \in C^{3}$. The assumptions on the boundary data may also be relaxed but this aspect shall be postponed for a further investigation.

7. Existence results. Various existence theorems, such as Corollary 1.2, may now be deduced from the estimates of $\S \S 2$ to 6 . We shall present separately the quasilinear and fully nonlinear cases as the existence theorems for the former only require $C^{1, \alpha}$ estimates. Moreover our first existence result below for the case of a quasilinear equation with a quasilinear boundary condition follows directly from the Leray-Schauder theorem, familiar from the corresponding Dirichlet problem. For the cases of quasilinear or fully nonlinear equations with general nonlinear boundary conditions, the nonlinear method of continuity or some related technique must be used to prove the basic existence theorems although the Leray-Schauder 
theorem can then be used to remove those hypotheses introduced for purely functional analytic reasons. Various of these problems with fully nonlinear boundary conditions will be discussed.

We begin by recalling the a priori solution bounds from [18, Lemmas 3.1, 3.2] which we formulate here for fully nonlinear equations. Throughout this section we shall assume that the boundary value problem

$$
F[u]=F\left(x, u, D u, D^{2} u\right)=0 \quad \text { in } \Omega, \quad G[u]=G(x, u, D u)=0 \quad \text { in } \partial \Omega
$$

is elliptic on $\Gamma$ and oblique on $\Gamma^{\prime}$ so that $\lambda$ and $\chi$ are positive wherever they are defined.

LEMMA 7.1. Let $\partial \Omega \in C^{2}$ and nonnegative $\rho \in C^{2}(\bar{\Omega})$ satisfy $D \rho=\gamma$ on $\partial \Omega$. If there are nonnegative constants $M_{0}$ and $m_{1}$ such that

$$
\begin{aligned}
& z F\left(x, z, D w, D^{2} w\right)<0 \\
& z G(x, z, D w)<0
\end{aligned}
$$

for all $|z| \geq M_{0}$ and $w=-(\operatorname{sign} z) m_{1} \rho$, then any solution of (7.1) obeys the estimate

$$
|u|_{0 ; \Omega} \leq M_{0}+m_{1}|\rho|_{0 ; \Omega} .
$$

PROOF. We only prove the upper bound for $u$; the lower bound is proved in a similar fashion. Set $v=v_{0}-m_{1} \rho$, where $v_{0}$ is a constant chosen so that $\sup (u-v)=0$, and let $x_{0}$ be a point in $\bar{\Omega}$ where $u=v$. If $x_{0} \in \partial \Omega$, then

$$
G\left(x_{0}, v_{0}-m_{1} \rho\left(x_{0}\right),-m_{1} \gamma\right)=G\left(x_{0}, u\left(x_{0}\right), D v\left(x_{0}\right)\right) \geq G\left(x_{0}, u\left(x_{0}\right), D u\left(x_{0}\right)\right)=0
$$

so we must have

$$
v_{0} \leq M_{0}+m_{1} \rho\left(x_{0}\right) \leq M_{0}+m_{1}|\rho|_{0}
$$

If $x_{0} \in \Omega$, we have

$$
\begin{gathered}
F\left(x_{0}, v_{0}-m_{1} \rho\left(x_{0}\right),-D w\left(x_{0}\right),-D^{2} w\left(x_{0}\right)\right)=F\left(x_{0}, u\left(x_{0}\right), D u\left(x_{0}\right),-m_{1} D^{2} \rho\left(x_{0}\right)\right) \\
\geq F\left(x_{0}, u\left(x_{0}\right), D u\left(x_{0}\right), D^{2} u\left(x_{0}\right)\right)=0,
\end{gathered}
$$

so that again $v_{0} \leq M_{0}+m_{1}|\rho|_{0}$. Combining these two cases with the obvious inequality $u \leq v_{0}$ completes the proof.

It is a simple matter to construct $\rho \in C^{2}(\bar{\Omega})$ with $D \rho=\gamma$ on $\partial \Omega$ whenever $\partial \Omega \in$ $C^{2}$. An interesting feature of the above proof is that no monotonicity assumptions with respect to $z$ are made on $F$ or $G$. Note also that (7.2) and (7.3) are modelled on the linear problem

$$
\Delta u-u=f(x) \quad \text { in } \Omega, \quad D u \cdot \gamma=g(x) \text { on } \partial \Omega .
$$

An estimate of $|u|_{0}$ based on the linear problem

$$
\Delta u=f(x) \text { in } \Omega, \quad D u \cdot \gamma-u=g(x) \text { on } \partial \Omega,
$$

is also possible. For this case we recall [18, Lemma 3.1] (in its obvious fully nonlinear form). 
LEMMA 7.2. Suppose there is a nonnegative constant $\mu_{1}$ such that

$$
(\operatorname{sign} z) F(x, z, p, 0) \leq \mu_{1} \lambda(1+|p|)
$$

for all $x, z, p \in \Omega \times \mathbf{R} \times \mathbf{R}^{n}$, and a nondecreasing function $M_{0}$ such that

$$
(\operatorname{sign} z) G<0 \text { for }|z| \geq M_{0}(|p|)
$$

and $(x, z, p) \in \Omega \times \mathbf{R} \times \mathbf{R}^{n}$. If $u \in C^{1}(\bar{\Omega}) \cap C^{2}(\Omega)$ is a solution of (7.1), then

$$
|u|_{0 ; \Omega} \leq C\left(\mu_{1}, M_{0}, \Omega\right)
$$

All of our existence results will be stated using Lemma 7.1, the corresponding formulations based on Lemma 7.2 being left to the reader. The following regularity result, taken from [23], will also be employed throughout this section.

LEMMA 7.3. Let $\partial \Omega \in C^{2, \alpha}$ for some $\alpha \in(0,1)$ and suppose that $F \in C^{1, \alpha}(\bar{\Gamma})$, $G \in C^{1, \alpha}\left(\Gamma^{\prime}\right)$. Then any solution $u \in C^{2}(\bar{\Omega})$ of (7.1) automatically lies in $C^{2, \alpha}(\bar{\Omega})$. For quasilinear $F$ we need only $F \in C^{0,1}(\bar{\Gamma})$.

Lemma 7.3 follows from the intermediate $C^{1, \alpha}(\bar{\Omega})$ estimates of $[\mathbf{2 1}]$ for solutions of linear problems with $C^{\alpha}(\bar{\Omega})$ coefficients in $C^{2, \alpha}$ domains. Let us now deal first with the quasilinear case.

Quasilinear equations. We first consider the quasilinear problem

$$
\begin{gathered}
Q[u]=a^{i j}(x, u, D u) D_{i j} u+a(x, u, D u)=0 \text { in } \Omega, \\
G(x, u, D u)=0 \text { on } \partial \Omega,
\end{gathered}
$$

for the special case when $G$ is also quasilinear, that is,

$$
G(x, z, p)=b(x, z) \cdot p+g(x, z),
$$

where $b^{i}$ and $g$ are real valued functions on $\partial \Omega \times \mathbf{R}$.

THEOREM 7.4. Let $\partial \Omega \in C^{2, \alpha}$ for some $\alpha \in(0,1)$ and $a^{i j}, a \in C^{1}\left(\bar{\Omega} \times \mathbf{R} \times \mathbf{R}^{n}\right)$, $b^{i}, g \in C^{1, \alpha}(\partial \Omega \times \mathbf{R})$ and suppose that the following structure conditions hold for all $(x, z, p) \in \Omega \times \mathbf{R} \times \mathbf{R}^{n}$, some positive function $\lambda$ on $\bar{\Omega} \times \mathbf{R} \times \mathbf{R}^{n}$ and nondecreasing functions $\mu, \mu_{0}, \mu_{1}$ on $\mathbf{R}$ :

$$
\begin{aligned}
& a^{i j} \xi_{i} \xi_{j} \geq \lambda|\xi|^{2} \quad \text { for all } \xi \in \mathbf{R}^{n} \\
& \left|a^{i j}\right| \leq \mu(|z|) \lambda ; \quad|a| \leq \mu_{0}(|z|) \lambda\left(1+|p|^{2}\right) \\
& (1+|p|)\left|a_{p}^{i j}\right|,\left|a_{z}^{i j}\right|,\left|a_{x}^{i j}\right| \leq \mu_{1}(|z|) \lambda ; \\
& (1+|p|)\left|a_{p}\right|,\left|a_{z}\right|,\left|a_{x}\right| \leq \mu_{1}(|z|) \lambda\left(1+|p|^{2}\right) .
\end{aligned}
$$

Suppose also that $b \cdot \gamma>0$ on $\partial \Omega \times \mathbf{R}$ and that (7.2), (7.3) hold. Then the problem (7.8), (7.9) has a solution $u \in C^{2, \alpha}(\bar{\Omega})$.

Proof. For $\delta \in(0,1)$ to be chosen, define a map $T: C^{1, \delta}(\bar{\Omega}) \times[0,1] \rightarrow C^{1, \delta}(\bar{\Omega})$ by letting $u=T(v, \sigma)$ be the unique $C^{2, c \delta}(\bar{\Omega})$ solution of the problem

$$
\begin{aligned}
& \sigma\left(a^{i j}(x, v, D v) D_{i j} u+a(x, v, D v)+v-u\right)+(1-\sigma)(\Delta u-u)=0 \quad \text { in } \Omega, \\
& b(x, v) \cdot D u+\sigma g(x, v)=0 \quad \text { on } \partial \Omega .
\end{aligned}
$$

It follows from [9, Theorem 6.31] that $T$ is well defined, that $u=T(u, \sigma)$ implies $u \in$ $C^{2, \alpha}(\bar{\Omega})$, and that $u=T(u, 1)$ if and only if $u$ is a $C^{2, \alpha}(\bar{\Omega})$ solution of $(7.8),(7.9)$. 
A standard argument, similar to the one in $[9, \S 11.4]$, shows that $T$ is compact, and the boundary point lemma implies that $T(v, 0)=0$ for all $v \in C^{1, \delta}(\bar{\Omega})$. Thus the Leray-Schauder fixed point theorem in the form $[9$, Theorem 11.6] reduces the solvability of $(7.8)$ to the establishment of uniform $C^{1, \delta}(\bar{\Omega})$ estimates for the fixed points of $T(\cdot, \sigma)$. These fixed points are all solutions of

$$
\begin{aligned}
& Q_{\sigma}[u] \equiv \sigma Q u+(1-\sigma)(\Delta u-u)=0 \quad \text { in } \Omega, \\
& G_{\sigma}[u] \equiv b(x, u) \cdot D u+\sigma g(x, u)=0 \quad \text { on } \partial \Omega,
\end{aligned}
$$

and $Q_{\sigma}, G_{\sigma}$ satisfy the hypotheses of Lemma 7.1 with the same $m_{1}$ as in the hypotheses of this theorem and $M_{0}$ replaced by $\max \left\{M_{0}, m_{1}|\Delta \rho|_{0}\right\}$. Next conditions (7.11) imply that F1 and F3 hold for $F=Q_{\sigma}$ while in addition the quasilinear structure gives F2. Also G2 and G3 are automatically satisfied for $G_{\sigma}$ because of the quasilinearity of the boundary condition. It therefore follows from Lemma 7.1 and Theorems 2.3,3.3, and 4.1 that any solution of $u=T(u, \sigma)$ obeys the estimate $|u|_{1, \delta ; \Omega} \leq C$ for some constants $C$ and $\delta \in(0,1)$ independent of $u$ and $\sigma$. Using this $\delta$ in the definition of $T$ completes the proof.

Note that (7.2) will hold for any positive $m_{1}$ if

$$
\lim _{|z| \rightarrow \infty}(\operatorname{sign} z) a(x, z, p) / \Lambda(x, z, p)=-\infty
$$

uniformly on bounded subsets of $\Omega \times \mathbf{R}^{n}$ while (7.3) will hold for the operator (7.9), for any positive $m_{1}$, if

$$
\lim _{|z| \rightarrow \infty}(\operatorname{sign} z) g(x, z) / b(x, z) \cdot \gamma \leq 0
$$

uniformly on $\partial \Omega$. Moreover we need only assume $a^{i j}, a \in C^{0,1}\left(\bar{\Omega} \times \mathbf{R} \times \mathbf{R}^{n}\right)$, and if also only $b, g \in C^{0,1}(\bar{\Omega} \times \mathbf{R})$, then a simple approximation argument yields a solution $u \in C^{1, \beta}(\bar{\Omega}) \cap C^{2, \beta}(\bar{\Omega})$ for all $\beta \in(0,1)$. When the boundary condition is not quasilinear, even if the differential equation is, the Leray-Schauder approach is no longer immediately applicable. In its place we use the nonlinear method of continuity [9, Theorem 17.30].

LEMMA 7.5. Let $\alpha, \delta \in(0,1)$ and $\partial \Omega \in C^{2, \alpha}$. Suppose that $a^{i j}, a$ $\in C^{1}\left(\bar{\Omega} \times \mathbf{R} \times \mathbf{R}^{n}\right), G \in C^{1, \alpha}\left(\partial \Omega \times \mathbf{R} \times \mathbf{R}^{n}\right), a_{p}^{i j}, a_{z}^{i j}, a_{p}, a_{z} \in C^{\delta}\left(\bar{\Omega} \times \mathbf{R} \times \mathbf{R}^{n}\right)$, $G_{p}, G_{z} \in C^{1, \delta}\left(\partial \Omega \times \mathbf{R} \times \mathbf{R}^{n}\right)$ and that (7.10) holds together with

$$
a_{z}^{i j}=0, \quad a_{z}<0 \quad \text { in } \Omega \times \mathbf{R} \times \mathbf{R}^{n}, \quad G_{z} \leq 0 \quad \text { on } \partial \Omega \times \mathbf{R} \times \mathbf{R}^{n} .
$$

Then, if for some $\psi \in C^{3}(\bar{\Omega})$ and some $\beta \in(0, \alpha)$ the set $E=\left\{u \in C^{2, \beta}(\bar{\Omega}) \mid\right.$ $Q[u]=\sigma Q[\psi]$ in $\Omega, G[u]=\sigma G[\psi]$ on $\partial \Omega$ for some $\sigma \in[0,1]\}$ is bounded in $C^{1}(\bar{\Omega})$, the boundary value problem (7.8) has a unique solution $u \in C^{2, \alpha}(\bar{\Omega})$.

Proof. From [9, Theorem 17.30] and Theorem 4.1 we obtain the unique solvability of (7.8) in $C^{2, \beta}(\bar{\Omega})$ and hence in $C^{2, \alpha}(\bar{\Omega})$ by virtue of Lemma 7.3.

The smoothness assumptions on $Q$ and $G$ may in practice be weakened through approximation (see also the discussion after Corollary 7.7). Functions $\psi$ can also generally be chosen to satisfy $G[\psi]=0$ on $\partial \Omega$ (see $[19$, Theorem 2]). We can now prove an existence theorem for quasilinear equations under the natural structure conditions. 
THEOREM 7.6. Let $\partial \Omega \in C^{2, \alpha}$ for some $\alpha \in(0,1)$. Suppose that $a^{i j}$, $a \in C^{1}\left(\bar{\Omega} \times \mathbf{R} \times \mathbf{R}^{n}\right)$ satisfy the natural conditions (7.10),(7.11) while $G \in$ $C^{1, \alpha}\left(\bar{\Omega} \times \mathbf{R} \times \mathbf{R}^{n}\right)$ satisfies $\mathrm{G} 2, \mathrm{G} 3$, that is

$$
\begin{aligned}
& \left|G\left(x, z, p^{\prime}\right)\right| \leq \mu_{0}(|z|) \chi\left(1+\left|p^{\prime}\right|\right) \quad\left(p^{\prime}=p-(p \cdot \gamma) \gamma\right) \\
& (1+|p|)\left|G_{p}\right|,\left|G_{z}\right|,\left|G_{x}\right| \leq \mu_{1}(|z|) \chi(1+|p|) .
\end{aligned}
$$

Then if (7.2), (7.3) hold for some $M_{0}$ and $m_{1}$, the boundary value problem (7.8) has a $C^{2, \alpha}(\bar{\Omega})$ solution.

ProOF. This time we define a map $T: C^{2}(\bar{\Omega}) \times[0,1] \rightarrow C^{2}(\bar{\Omega})$ by letting $u=T(v, \tau)$ be the unique $C^{2, \alpha}(\bar{\Omega})$ solution of

$$
\begin{aligned}
& \tau\left(a^{i j}(x, v, D v) D_{i j} u+a(x, v, D v)+v-u\right)+(1-\tau)(\Delta u-u)=0 \quad \text { in } \Omega, \\
& \tau(G(x, v, D u)+v-u)+(1-\tau) D u \cdot \gamma=0 \quad \text { on } \partial \Omega .
\end{aligned}
$$

We must first show that $T$ is well defined. Assuming temporarily that $G$ satisfies the smoothness conditions of Lemma 7.4, we apply Lemma 7.4 to (7.14) with $\psi \equiv 0$ and fixed $(v, \tau) \in C^{2}(\bar{\Omega}) \times[0,1)$. The set $E$ corresponding to (7.14) is bounded in $C^{1}(\bar{\Omega})$ by virtue of Lemma 7.1 and Theorems $2.3,3.3$, because $\lambda>1-\tau$. Hence $T$ is well defined on $C^{2}(\bar{\Omega}) \times[0,1)$. To see that $T$ is well defined for $\tau=1$, we observe that (7.14) has at most one solution if $\tau \in[0,1]$ and that for fixed $v$, the functions $T(v, \tau)$ are uniformly bounded in $C^{2, \alpha}(\bar{\Omega})$ for $\tau \in[0,1]$ and hence $T(v, 1)=\lim _{\tau \rightarrow 1} T(v, \tau)$. It then follows from Lemmas 7.1, 7.3 and Theorems 2.3, 3.3, and 4.1 that $T$ is compact, and an argument similar to that in Theorem 7.4 completes the proof.

By further approximation of the boundary function $G$ (for example by means of mollification), we may further reduce the smoothness of $G$ in Theorem 7.6.

COROLLARY 7.7. Suppose all the hypotheses of Theorem 7.6 hold except that $G$ is only assumed in $C^{0,1}\left(\bar{\Omega} \times \mathbf{R} \times \mathbf{R}^{n}\right.$ ) (with (7.13) holding for the weak derivatives of $G)$. Then the boundary value problem $(7.8)$ has a $C^{1, \beta}(\bar{\Omega}) \cap C^{2}(\Omega)$ solution for some $\beta>0$.

More generally we can conclude, in the absence of conditions (7.2), (7.3) in Theorem 7.6 and Corollary 7.2, that the problem (7.8) is classically solvable if the family of fixed points of the problems $(7.14)$ is a priori bounded in $C^{0}(\bar{\Omega})$. Other families may also be substituted. An important feature of Theorem 7.6 is its applicability to problems which are not uniquely solvable (cf. [9, Chapter 17; 14, Chapter $10 ; 16,19]$, etc.). For example, let $f \in C^{1}(\mathbf{R})$ and $g \in C^{1, \alpha}(\mathbf{R})$ satisfy $f(z)=g(z)=0$ for $|z| \leq 1,-f(z)=g(z)=z /|z|$ for $|z| \geq 2$. Then all the hypotheses of Theorem 7.6 are satisfied for the problem

$$
\Delta u+f(u)=0 \quad \text { in } \Omega, \quad D u \cdot \gamma+g(u)=0 \quad \text { on } \partial \Omega
$$

provided $\partial \Omega \in C^{2, \alpha}$, and any constant in the interval $[-1,1]$ solves this problem.

Note that Theorem 7.6 extends Theorem 7.4. Also Theorem 7.6 includes [18, Theorem 2] provided we rewrite conditions (7.11) in terms of the operators $\delta$ and $\delta^{\prime}$ from $\S 3$. As a special example we consider the problem

$Q[u]=\Delta u-u=0 \quad$ in $\Omega, \quad G[u]=D u \cdot \gamma+g(x, u)\left(1+|D u|^{2}\right)^{1 / 2}=0 \quad$ on $\partial \Omega$. 
A straightforward calculation shows that the hypotheses of Theorem 7.6 are satisfied if $g \in C^{\mathbf{1 , \alpha}}(\partial \Omega \times \mathbf{R})$ and

$$
\sup _{\partial \Omega} \lim _{|z| \rightarrow \infty} g(x, z) \operatorname{sign} z<1 .
$$

Using [18] we would need more smoothness of $g, \partial \Omega$, and, more significantly, the inequality $g_{z} \leq 0$.

Before moving on to fully nonlinear equations we digress to point out that the conditions $G_{z}, G_{p} \in C^{1, \delta}\left(\bar{\Omega} \times \mathbf{R} \times \mathbf{R}^{n}\right)$ in the statement of Lemma 7.5 may be removed at the outset by applying the method of continuity argument with the weighted Hölder space $H_{2+\beta}^{(-1-\beta)}(\Omega)$ from [8] in place of $C^{2, \beta}(\bar{\Omega})$ and the intermediate Schauder theory of $[\mathbf{2 1}]$ in place of $[9$, Theorem 6.31]. A key observation here is that a solution $u \in H_{2+\beta}^{(-1-\beta)}(\Omega)$ of $Q[u]=\sigma Q[u], G[u]=\sigma G[\psi]$ will lie in $C^{2, \beta}(\bar{\Omega})$. The weighted Hölder spaces can also be used to replace the assumption $\psi \in C^{3}(\bar{\Omega})$ with $\psi \in H_{3}^{(-2-\beta)}$, in which case [8, Theorem 2.5 and 19, Theorem 2] shows that $\psi$ can be chosen to satisfy $G[\psi]=0$.

Fully nonlinear equations. For fully nonlinear equations, we combine the method of continuity [9, Theorem 17.28], Theorem 6.3 and Lemma 2.3 to obtain the following result.

LEMMA 7.8. Let $\partial \Omega \in C^{4}$ and suppose that for some $\alpha \in(0,1)$, we have $F \in C^{2, \alpha}(\bar{\Gamma}), G \in C^{3, \alpha}\left(\Gamma^{\prime}\right)$ with $F$ concave with respect to the $r$ variables. Suppose also that $\lambda$ extends to a positive function on $\bar{\Gamma}$ and

$$
F_{z}<0 \text { on } \Gamma, \quad G_{z} \leq 0 \text { on } \Gamma^{\prime} .
$$

Then if for some $\psi \in C^{4}(\bar{\Omega})$ the set

$E=\left\{u \in C^{2, \alpha}(\bar{\Omega}) \mid F[u]=\sigma F[\psi]\right.$ in $\Omega, G[u]=\sigma G[\psi]$ on $\partial \Omega$ for some $\left.\sigma \in[0,1]\right\}$

is bounded in $C^{2}(\bar{\Omega})$, the problem

$$
F[u]=0 \quad \text { in } \Omega, \quad G[u]=0 \quad \text { on } \partial \Omega
$$

has a unique solution $u \in C^{2, \alpha}(\bar{\Omega})$.

Standard linear theory improves the solution in Lemma 7.8 to lie in $C^{3, \delta}(\bar{\Omega}) \cap$ $C^{4, \alpha}(\Omega)$ for all $\delta \in(0,1)$ and moreover $\partial \Omega \in C^{4, \alpha}$ implies $u \in C^{4, \alpha}(\bar{\Omega})$. Although the hypotheses of Lemma 7.8 are easily weakened to conform with those in Lemma 7.4 (e.g. $\partial \Omega \in C^{3, \alpha}, F \in C^{2}, F_{r}, F_{p}, F_{z} \in C^{1, \alpha}$ ), this greater generality makes the statements of resuslts much more cumbersome. Therefore we leave it to the reader to supply refined versions of our theorems. We are now in a position to prove Corollary 1.2.

THEOREM 7.9. Let $\partial \Omega \in C^{4}, F \in C^{2}(\Gamma), G \in C^{2}\left(\Gamma^{\prime}\right)$ and suppose that $F$ satisfies the structure conditions $\mathrm{F} 1-\mathrm{F} 5$, that is

$$
\begin{aligned}
& \Lambda \leq \mu \lambda, \quad|F(x, z, p, 0)| \leq \mu_{0} \lambda\left(1+|p|^{2}\right), \\
& \left|F_{p}\right|,\left|F_{z}\right|,\left|F_{x}\right| \leq \mu_{1} \lambda\left(1+|p|^{2}+|r|\right), \\
& \left|F_{r p}\right|,\left|F_{r z}\right|,\left|F_{r x}\right| \leq \mu_{2} \lambda, \\
& \left|F_{p p}\right|,\left|F_{p z}\right|,\left|F_{p x}\right|,\left|F_{z z}\right|,\left|F_{z x}\right|,\left|F_{x x}\right| \leq \mu_{2} \lambda(1+|r|), \quad F_{r r} \leq 0,
\end{aligned}
$$


in $\Gamma$, where $\mu_{0}=\mu_{0}(|z|), \mu_{1}=\mu_{1}(|z|), \mu_{2}=\mu_{2}(|z|+|p|)$ are nondecreasing, and $G$ satisfies $\mathrm{G} 2, \mathrm{G} 3$, that is (7.13).

Suppose also that

$$
\sup _{\Gamma} F_{z}<0, \quad \sup _{\Gamma^{\prime}} G_{z} \leq 0
$$

Then the boundary value problem (7.17) has a unique solution $u \in C^{2, \alpha}(\bar{\Omega})$ for all $\alpha \in(0,1)$.

Proof. When $F \in C^{2, \alpha}, G \in C^{3, \alpha}$, and $\lambda, \chi$ are bounded away from zero, the result follows easily from Theorems $2.3,3.3,4.1,5.3,6.3$ and Lemmas 7.1 and 7.8. The general case may then be achieved through approximation by boundary values of the form

$$
F_{\varepsilon}[u]+\varepsilon \Delta u=0 \quad \text { in } \Omega_{\varepsilon}, \quad G_{\varepsilon}[u]+\varepsilon D u \cdot \gamma=0 \text { on } \partial \Omega_{\varepsilon}
$$

for sufficiently small $\varepsilon>0$, where $\Omega_{\varepsilon}=\{x \in \Omega \mid d(x)>\varepsilon\}$ and $F_{\varepsilon}$ and $G_{\varepsilon}$ are appropriate mollifications of $F$ and $G$ on $\Omega \times \mathbf{R} \times \mathbf{R}^{n} \times \mathbf{S}^{n}$ and $\Omega \times \mathbf{R} \times \mathbf{R}^{n}$, respectively.

Next we observe that the condition (7.21) can be relaxed through a procedure analogous to that in Theorem 7.6 with, instead of (7.14), the problems

$$
\begin{aligned}
& \tau\left\{F\left(x, v, D v, D^{2} u\right)+v-u\right\}+(1-\tau)(\Delta u-u)=0 \text { in } \Omega, \\
& \tau\{G(x, v, D u)+v-u\}+(1-\tau) D u \cdot \gamma=0 \text { on } \partial \Omega .
\end{aligned}
$$

We thus obtain the following generalization of Theorem 7.9.

COROLlaRY 7.10. Let $\partial \Omega \in C^{4}, F \in C^{2}(\Gamma), G \in C^{2}\left(\Gamma^{\prime}\right)$ and suppose that (7.13), (7.18), (7.19), (7.20) are satisfied and that (7.2), (7.3) hold for $|z| \geq M_{0}$ and some $m_{1} \geq 0$. Then problem (7.17) has a solution $u \in C^{2, \alpha}(\bar{\Omega})$ for all $\alpha<1$.

Our estimates are also applicable to problems involving Bellman operators although in this case we cannot make assertions on the regularity of solutions as strongly as before.

THEOREM 7.11. Let $\partial \Omega \in C^{4}$, let $\left\{F_{k}\right\}$ be a sequence of operators with $F_{k} \in$ $C^{2}(\Gamma)$ for each $k$ and let $G \in C^{0,1}\left(\bar{\Omega} \times \mathbf{R} \times \mathbf{R}^{n}\right)$. Suppose that (7.13), (7.18), (7.19), (7.20) hold for $F_{k}, G$ with $\mu_{1} \mu_{0}, \mu_{1}, \mu_{2}$ independent of $k$ and also

$$
\sup _{\Gamma, k} F_{k, z}<0, \quad \sup _{\Gamma^{\prime}} G_{z} \leq 0 .
$$

Then the problem

$$
F[u]=\inf _{k} F_{k}[u]=0 \quad \text { in } \Omega, \quad G[u]=0 \quad \text { on } \partial \Omega
$$

has a unique solution $u \in C^{2, \alpha}(\Omega) \cap C^{1, \alpha}(\bar{\Omega})$ for some $\alpha \in(0,1)$. Moreover if $G \in C^{1,1}\left(\bar{\Omega} \times \mathbf{R} \times \mathbf{R}^{n}\right)$, then $u \in C^{1,1}(\bar{\Omega})$.

PROOF. The operator $F$ in (7.24) may be approximated through mollification as in the case of the Dirichlet problem $[9, \S 17.5]$ and the operator $G$ approximated as for Corollary 7.7. The first assertion of the theorem then follows from Theorems 2.3, 3.3, 4.1 and the interior estimates in Theorem 6.2 while the second assertion depends also on Theorem 5.4. 
We remark that the condition on $\partial \Omega$ in Theorem 7.11 can be relaxed by approximation to $\partial \Omega \in C^{2, \beta}$ for some $\beta \in(0,1)$ (with $\partial \Omega \in C^{3, \beta}$ to imply $u \in C^{1,1}(\bar{\Omega})$ ). The conditions (7.23) may also be generalized as in Corollary 7.10, with a possible loss of uniqueness. It is also interesting to note that the boundary condition $G$ in Corollaries 7.7, 7.10 embraces Bellman type conditions of the form

$$
G[u]=\inf _{k}\left(\text { or } \sup _{k}\right) G_{k}[u]=0 \text { on } \partial \Omega,
$$

where $G_{k} \in C^{2}\left(\Gamma^{\prime}\right)$ satisfy (7.13), (7.23) uniformly in $k$.

For equations in two variables, our remarks at the end of $\S \S 4,5$, and 6 show that condition (7.20) can be dispensed with in Theorem 7.9, Corollary 7.10, and Theorem 7.11 with the operators $F, F_{k}$ only required to lie in $C^{0,1}(\bar{\Gamma})$.

Finally we point out that the existence Theorems $7.9,7.11$ themselves can be used to relax global smoothness hypotheses in our estimates through appropriate modification of the boundary value problems which cause the given solution $u$ to become unique. In particular we find that Theorem 4.1 holds for $u \in C^{1}(\bar{\Omega}) \cap C^{2}(\Omega)$ whence $u \in C^{1, \alpha}(\bar{\Omega})$ and by virtue of Lemma 5.1 ,

$$
\sup _{x \in \Omega} d_{x}^{\alpha-1}\left|D^{2} u(x)\right|<\infty .
$$

By modifying the proofs of Lemmas 5.2, 5.3 (similarly to Lemma 2.2) we then deduce Theorem 5.4 also for $u \in C^{1}(\bar{\Omega}) \cap C^{2}(\Omega)$. In this case we may create a new boundary value problem with unique solution $u$ by suitable truncation of $G$ and replacement of $F$ by

$$
\tilde{F}=F(x, u(x), p, r)+A(u(x)-z)
$$

for sufficiently large $A$. As a result, Theorem 1.1 will be valid for arbitrary classical solutions.

Supplementary remarks (January, 1986). Since this paper was submitted for publication in 1984, there have appeared further developments $[39,40]$ which lead to improvements of our second derivative estimates and the consequent existence theorems for fully nonlinear equations. In particular it was shown by Trudinger [40] that condition (6.3) is not necessary in Lemma 6.1 and Theorem 6.2 so that the global estimate $(6.10)$ is independent of $\mu_{3}$. It then follows that the second derivative bounds in Theorem 5.4 may be alternatively deduced through the interpolation argument of $[\mathbf{3 0}, \mathbf{4 1}]$ and moreover that if $G \in C^{1,1}\left(\bar{\Omega} \times \mathbf{R} \times \mathbf{R}^{n}\right)$ in the Bellman existence result, Theorem 7.11, then the solution $u \in C^{2, \alpha}(\bar{\Omega})$ for some $\alpha>0$, depending only on $n$ and $\mu$. But the hypotheses of these results may be even further reduced by adaptation of the perturbation argument invoked by Safonov [39] for the Bellman Dirichlet problem. As a result, the condition (6.2) in Theorem 6.2 (and subsequently, by interpolation, condition (5.4) in Theorem 5.4) may be replaced simply by the concavity of $F$ with respect to $r$. This shows that the natural condition F4 can be dispensed with altogether, both here and in the Dirichlet problem [30]. Also the assumed boundary smoothness can be relaxed to $\partial \Omega \in C^{2, \beta}, G \in C^{1, \beta}\left(\bar{\Omega} \times \mathbf{R} \times \mathbf{R}^{n}\right)$ for some $\beta>0$, with the Hölder exponent $\eta$ in Theorem 6.2 depending additionally on $\beta$. Taking account of these developments, we see that in our final existence result, Theorem 7.11, we need only 
assume $\partial \Omega \in C^{2, \beta}$ for some $\beta>0$ with the structure condition (7.20) reduced to the concavity condition, $F_{r r} \leq 0$ and when $G \in C^{1, \beta}\left(\bar{\Omega} \times \mathbf{R} \times \mathbf{R}^{n}\right)$, the solution $u \in C^{2, \alpha}(\bar{\Omega})$. Further details will appear in the lecture notes [42].

To conclude these remarks we also mention related recent work of Lieberman [35] and Lieberman and Korevaar [34] on gradient bounds for nonuniformly elliptic quasilinear equations, Nadirashvili [38] on Hölder estimates and Madjarova [37] and Lieberman [36] on the two variable case. Also despite the fact that our techniques in $\S 5$ can be replaced by interpolation, they are nevertheless important as they extend to embrace nonuniformly elliptic equations or obstacle problems where second derivative Hölder estimates are not feasible; (see for example [40]).

\section{REFERENCES}

1. S. Agmon, A. Douglis and L. Nirenberg, Estimates near the boundary for solutions of elliptic partial differential equations satisfying general boundary conditions. I. Comm. Pure Appl. Math. 12 (1959), 623-727.

2. L. Caffarelli, L. Nirenberg and J. Spruck, The Dirichlet problem for nonlinear second order elliptic equations. I. Monge-Ampère equations, Comm. Pure Appl. Math. 37 (1984), 369-402; II (with J. J. Kohn) Complex Monge-Ampère and uniformly elliptic equations, Comm. Pure Appl. Math. 38 (1985), 209-252.

3. L. C. Evans, Classical solutions of fully nonlinear, convex second order elliptic equations, Comm. Pure Appl. Math. 35 (1982), 333-363.

4. L. C. Evans and A. Friedman, Optimal stochastic switching and the Dirichlet problem for the Bellman equation, Trans. Amer. Math. Soc. 253 (1979), 365-389.

5. L. C. Evans and P.-L. Lions, Résolution des équations de Hamilton-Jacobi-Bellman pour des opérateurs uniformément elliptiques, C. R. Acad. Sci. Paris 290 (1980), 1049-1052.

6. R. Fiorenza, Sui problemi di derivata obliqua per le equazioni ellitiche quasi lineare, Ricerche Mat. 15 (1966), 74-108.

7. C. Gerhardt, Boundary value problems for surfaces of prescribed mean curvature, J. Math. Pures Appl. 58 (1979), 75-109.

8. D. Gilbarg and L. Hörmander, Intermediate Schauder estimates, Arch. Rational Mech. Anal. 74 (1980), 297-318.

9. D. Gilbarg and N. S. Trudinger, Elliptic partial differential equations of second order, 2nd ed., Springer-Verlag, Berlin, Heidelberg, New York and Tokyo, 1983.

10. L. I. Kamynin and B. N. Khimchenko, The principle of the maximum and boundary Lipschitz bounds for the solution of a second order elliptic-parabolic equation, Sibirsk. Mat. Zh. 15 (1974), 343-367; English transl., Siberian Math. J. 15 (1974), 242-260.

11. N. V. Krylov, Boundedly nonhomogeneous elliptic and parabolic equations, Izv. Akad. Nauk SSSR 46 (1982), 487-523; English transl., Math. USSR Izv. 20 (1983), 459-493.

12. __ Boundedly nonhomogeneous elliptic and parabolic equations in a domain, Izv. Akad. Nauk SSSR 47 (1983), 75-108; English transl., Math. USSR Izv. 21 (1984), 67-98.

13. N. V. Krylov and M. V. Safonov, Certain properties of solutions of paraboflic equations with measurable coefficients, Izv. Akad. Nauk SSSR 40 (1980), 161-175; English transl., Math. USSR Izv. 16 (1981), 151-164.

14. O. A. Ladyzhenskaya and N. N. Ural'tseva, Linear and quasilinear elliptic equations, Izdat. "Nauka", Moscow, 1964; English transl., Academic Press, New York, 1968; 2nd Russian ed., 1973.

15. G. M. Lieberman, The quasilinear Dirichlet problem with decreased regularity at the boundary, Comm. Partial Differential Equations 6 (1981), 437-497.

16. Solvability of quasilinear elliptic equations with nonlinear boundary conditions, Trans. Amer. Math. Soc. 273 (1982), 753-765.

17. $\ldots$, The conormal derivative problem for elliptic equations of variational type, J. Differential Equations 49 (1983), 218-257.

18. _ The nonlinear oblique derivative problem for quasilinear elliptic equations, Nonlinear Anal. 8 (1984), 49-65. 
19. II, J. Funct. Anal. 56 (1984), 210-219.

20. __ Regularized distance and its applications, Pacific J. Math. 117 (1985), 329-353.

21. __ Intermediate Schauder estimates for oblique derivative problems, Arch. Rational Mech. Anal. (to appear).

22. _ , The Dirichlet problem for quasilinear elliptic equations with continuously differentiable boundary values, Comm. Partial Differential Equations 11 (1986), 167-229.

23. G. M. Lieberman, Regularity of solutions of nonlinear elliptic boundary value problems, J. Reine Angew. Math. (to appear).

24. P.-L. Lions, Résolution de problèmes elliptiques quasilinéaires, Arch. Rational Mech. Anal. 74 (1980), 335-353.

25. $\_$, Résolution analytique des problèmes de Bellman-Dirichlet, Acta Math. 146 (1981), 151-166.

26. __ Hamilton-Jacobi-Bellman equations and the optimal control of stochastic systems, Proc. Internat. Congress Math. Warsaw, 1983.

27. P.-L. Lions and N. S. Trudinger, Neumann problems for uniformly elliptic Bellman equations, Math. Z. 191 (1985), 1-15.

28. L. M. Simon and J. Spruck, Existence and regularity of a capillary surface with prescribed contact angle, Arch. Rational Mech. Anal. 61 (1976), 19-34.

29. N. S. Trudinger, Local estimates for subsolutions and supersolutions of general second order elliptic quasilinear equations, Invent. Math. 61 (1980), 67-79.

30. , Fully nonlinear, uniformly elliptic equations under natural structure conditions, Trans. Amer. Math. Soc. 278 (1983), 751-769.

31. _ Regularity of solutions of fully nonlinear elliptic equations, Boll. Un. Mat. Ital. (6) 3-A (1984), 421-430.

32. N. N. Ural'tseva, Solvability of the capillary problem, Vestnik Leningrad. Univ. 19 (1973), 54-64; English transl., Vestnik Leningrad Univ. Math. 6 (1979), 363-375.

33. W. von Wahl, Über quasilineare ellitiche Differentialgleichungen in der Ebene, Manuscripta Math. 8 (1973), 59-67.

\section{SUPPLEMENTARY REFERENCES}

34. N. Korevaar and G. M. Lieberman, Gradient estimates for the capillary problem via the maximum principle (to appear).

35. G. M. Lieberman, Gradient bounds for solutions of non-uniformly elliptic oblique derivative problems, Nonlinear Anal. (to appear).

36. __ Two dimensional nonlinear boundary value problems for elliptic equations, Trans. Amer. Math. Soc. (to appear).

37. J. D. Madjarova, On the Neumann problem for a fully nonlinear convex elliptic equation, C. R. Acad. Bulgare Sci. 38 (1985), 183-186.

38. N. S. Nadirashvili, On a problem with oblique derivative, Mat. Sb. 127 (1985), 398-416.

39. M. V. Safonov, On the classical solution of Bellman's elliptic equation, Soviet Math. Dokl. 30 (1984), 482-485.

40. N. S. Trudinger, Boundary value problems for fully nonlinear elliptic equations, Proc. Centre for Math. Anal. Austral. Nat. Univ. 8 (1984), 65-83.

41. __ On an interpolation inequality and its application to nonlinear elliptic equations, Proc. Amer. Math. Soc. 95 (1985), 73-78.

42. L_ Lectures on nonlinear second order elliptic equations, Nankai Institute of Mathematics, Tiajin, China, 1985.

Department of Mathematics, IOWA State University, Ames, IOWA 50111

Centre for mathematical analysis, The Australian National University, G.P.O. BoX 4, CANBERRA, A.C.T. 2601, Australia 